\title{
The Fraternal Birth-Order Effect as Statistical Artefact: Convergent Evidence from Probability Calculus, Simulated Data, and Multiverse Meta- Analysis.
}

\author{
Johannes K. Vilsmeier¹, Michael Kossmeier ${ }^{1}$, Martin Voracek ${ }^{1}$, Ulrich S. \\ $\operatorname{Tran}^{1}$
}

${ }^{1}$ Department of Cognition, Emotion, and Methods in Psychology, Faculty of Psychology, University of Vienna, Liebiggasse 5, A-1010 Vienna, Austria

Keywords: Fraternal Birth-Order Effect, Sexual Orientation, Maternal Immune Hypothesis, SpecificationCurve and Multiverse Meta-Analysis, Simulation

\section{Summary}

For a quarter of a century researchers investigating the origins of sexual orientation have largely ascribed to the fraternal birth order effect (FBOE) as a fact, holding that older brothers increase the odds of homosexual orientation among men through an immunoreactivity process. Here, we triangulate the empirical foundations of the FBOE from three distinct, informative perspectives: First, drawing on basic probability calculus, we deduce mathematically that the body of statistical evidence of the FBOE rests on the false assumptions that effects of family size should be controlled for and that this could be achieved through the use of ratio variables. Second, using a data-simulation approach, we demonstrate that by using ratio variables, researchers are bound to falsely declare corroborating evidence of an excess of older brothers at a rate of up to $100 \%$, and that valid approaches attempting to quantify a potential excess of older brothers among homosexual men must control for the confounding effects of the number of older siblings. And third, we re-examine the empirical evidence of the FBOE by using a novel specification-curve and multiverse approach to meta-analysis. This yielded highly inconsistent and moreover similarly-sized effects across 64 male and 17 female samples $(N=2,778,998)$, compatible with an excess as well as with a lack of older brothers in both groups, thus, suggesting that almost no variation in the number of older brothers in men is attributable to sexual orientation.

\section{Introduction}

It has been a quarter of a century now since Blanchard and Bogaert [1,2] first reported that the number of older brothers is positively correlated with the odds of homosexual orientation in men, a phenomenon, which at the time was termed the fraternal-birth order effect (FBOE). Since then, well over 50 similar reports (see Part III below) have been interpreted as confirming Blanchard and Bogaert's [2] initial findings, and researchers investigating the origins of human sexual orientation seem to accept an excess of older brothers among homosexual men as a fact [3-5], even going as far as describing it as "the most consistent biodemographic correlate of sexual orientation in men" [6]. The accommodation of the FBOE seems paramount to any theory striving to account for the observed variation in human sexual orientation [3]. It seems that initially, the term FBOE denoted solely the observation of an excess of older brothers in homosexual men. Yet, over time, its meaning has changed and acquired a causal flavour, as is evident from the most recent definition of the FBOE put forth by Blanchard [5]: "Older brothers increase the odds of homosexuality of later-born males, whereas older sisters, younger brothers and youngers sisters have no effect on those odds." It is difficult to read this definition as merely a correlational statement, which is why from this point forward, we distinguish between the FBOE as a causal claim (older brothers increase the odds of homosexual orientation) and the observed excess of older brothers as merely an observational statement. Moreover, the FBOE implies an observed excess of older brothers in homosexual men, whereas the reverse does not follow logically. It thus seems natural to infer that observing an excess of older brothers in homosexual men raises the plausibility of the FBOE. However, other explanations involving a third variable (or any number of variables) underlying both homosexual orientation and a surplus of older brothers could be held as equally plausible.

Numerous sources report an estimated increase in the odds of homosexual orientation of approximately $33 \%$ per older brother [1,5,7], with a recent publication on the effect [8] purporting a range of $30-40 \%$. There are at least three additional claims associated with the FBOE. First, accepted as the most plausible 
biological account for the manner in which older brothers increase the odds of homosexual orientation in later-born males, the so-called maternal immune hypothesis $(\mathrm{MIH})$ [9-11] invokes maternal immunoreactivity as underlying the FBOE. It is hypothesised that Y-linked proteins of XY-male foetuses may enter the maternal system prenatally or perinatally. The maternal immune system supposedly reacts to this "alien" tissue by producing antibodies. These antibodies are released, once Y-linked proteins originating from subsequent XY-male foetuses are detected. Further, these antibodies may enter the circulation of the foetus, where they are hypothesised to modulate the proliferation of "sex-dimorphic brain regions", thus contributing to the development of homosexual orientation. Second, homosexual men should not only exhibit an excess of older brothers, but also a smaller excess of older sisters [8]. This necessarily smaller excess of older sisters seems to have been borne out of an observed excess of older sisters in homosexual men [8,12], which prompted the formulation of an auxiliary to the MIH, purporting that mothers of homosexual men must have had more miscarriages of XY-male foetuses as opposed to mothers of heterosexual men, and thus greater exposure to Y-linked proteins $[8,12]$. Third, no (comparable) association between the number of older brothers and the odds of homosexual orientation should be observable among women (e.g., [10]). Since XX-female foetuses do not have Y chromosomes, there are no Y-linked proteins which could enter the maternal system and elicit an immune response. Arguably, a similarly-sized effect in women would be incompatible with the current formulation of the MIH. To our knowledge, only a single study addressing the MIH directly has been published so far [11] and only a small number of studies investigating a potential excess of older brothers in homosexual vs. heterosexual women are available (see Part III below). Furthermore, only a few studies (see references in Part III below) provided direct estimates for the purported increase in the odds of homosexual orientation per older brother. Most other studies assessed whether homosexual men showed an excess of older brothers, as compared to heterosexual men.

In addition to the collection of primary studies, there are by now seven meta-analyses of this research literature, [8], every single one of which concluded that homosexual men showed a consistent excess of older brothers and by extension corroborated the FBOE. While all of these meta-analyses were conducted by the same group of researchers, they differ considerably with respect to their primary goals stated, the subsets of included samples or studies, and the type of meta-analytic model fitted to the data. An overview of these previous meta-analyses can be found in Table 1. Below, we focus primarily on the fourth [5], fifth [13], and seventh [8] of these meta-analyses for the following two reasons: first, there is a substantial overlap of the samples included in the different meta-analyses listed in Table 1, with a total of 54 unique samples. The three meta-analyses we focus on included a total of 45 of these unique samples. Second, and most relevant to the discussion in Part I below, these three meta-analyses employed a common effect-size metric, namely the older brothers odds ratio (OBOR; Table 2), whilst two of the existing seven metaanalyses did not employ any effect size at all $[7,15]$. Moreover, the set of samples comprising the observations for the remaining two meta-analyses $[14,16]$ was fully contained within (i.e., merely representing a subset of) the combined set of samples considered in the meta-analyses [5], [17], and [8]. We review and evaluate the first [14], second [7], third [15], and sixth [18] meta-analyses in the supplementary materials (S1).

We summarise the theoretical network of the FBOE and the associated claims involving the MIH as follows: All of the evidence in favour of the FBOE is based on the observation that homosexual men show a consistent excess of older brothers. Conditional on the FBOE being true, the MIH is invoked as a biological explanation for the FBOE, and the current formulation of the MIH holds that no comparable excess of older brothers among homosexual women should be observable. Suppose it turned out that an excess of older brothers among men was much more inconsistent than previously believed by researchers, due to the previous evidence for such an excess being due to misapplications of statistical methods. Suppose further that there was a similarly-sized excess of older brothers among homosexual women. These observations combined would strip the FBOE and the MIH of its verisimilitude. In what follows, we provide converging evidence for both of these suppositions.

This work consists of three parts, each considering the empirical foundations of the FBOE and the MIH from a different angle: In Part I, we show mathematically that currently recommended effect sizes and variable transformations $[5,18,19]$, which together are referred to as measures, all are falsely advertised as quantifying the putative excess of older brothers among homosexual men. Moreover, the shortcomings of these measures are based on the unfounded claims that (a) statistical models investigating the excess of older brothers must control for a confounding effect of overall family size $[5,19]$ and that $(b)$ this could be 
achieved through the use of ratio variables. As a result, existing inferential claims about the FBOE and the MIH based on these measures are methodological artefacts and thus spurious. In Part II, we illustrate the inadequacy of these measures using simulated data. We show, that in combination with a ritualistic application of null-hypothesis significance testing (NHST; e.g., [20]), researchers are bound to frequently draw incorrect conclusions about the presence of an excess of older brothers but not sisters at a rate much greater than the pre-experimentally chosen acceptable false-positive rate (i.e., the threshold for statistical significance). Having shown that the OBOR and related measures are incapable of quantifying a genuine excess of older brothers, we conduct a new meta-analysis (the eighth one about the FBOE literature) on the excess of older brothers in homosexual men. More precisely, in Part III, we present re-analyses of the three previous meta-analyses, which employed the OBOR and provide a glimpse at the multiverse of possible meta-analyses about this literature, using a specification-curve meta-analytic approach [21-23]. In addition, we present the first set of meta-analyses on a putative excess of older brothers among homosexual vs. heterosexual women, as well as meta-analytic syntheses on the difference between men and women regarding the magnitude of this effect. The results of Part III converge with the key findings from Parts I and II, in that there appears to be little, if any, empirical foundation in the FBOE and the MIH.

\section{Part I. How not to measure the excess of older brothers: Insights from probability calculus}

\subsection{Notation}

The most widely used effect size for quantifying the excess of older brothers among homosexual men, the $O B O R$, is implicitly defined at the level of siblings, as reported by study participants. We thus need to introduce some notation to be able to show how precisely the OBOR fails to address the effect of interest.

If the reported siblings are regarded as the sample, the following events can be defined: A given sibling can be either an older brother, an older sister, a younger brother or a younger sister of the study participant who reported him or her. These events are denoted by OB, OS, YB and YS respectively. The complement of any event is denoted by the superscript $c$ (as in "complement"). For instance, $\mathrm{OB}^{c}$ denotes the event that a given sibling is either an older sister, a younger brother, or a younger sister. The event $\mathrm{OB}^{c}$ will mostly be referred to as Other, meaning that the sibling is not an older brother but any of the other three possible sibling types. There are instances in this work, where Other refers to siblings who are not older sisters, which should be clear from the given context. Furthermore, we denote the event that a given sibling is an older sibling (i.e., the union of $\mathrm{OB}$ and OS) by Older. Its complement Older $^{c}$ denotes the event that a given sibling is a younger sibling (for the sake of simplicity, twins, triplets, etc. are not accounted for).

A sibling can either be reported by a homosexual or by a heterosexual study participant. These events are denoted by Hom and Het, respectively, and are regarded as complementary. To aid readability, we use Het instead of $\mathrm{Hom}^{c}$, as the events Hom and Het are extensively referred to in the following.

The probability that an event $\mathrm{A}$ occurs is denoted by $\mathrm{P}(\mathrm{A})$. For instance, $\mathrm{P}(\mathrm{OB})$ should be read as "the probability that a given sibling is an older brother to the participant who reported him or her". The probability of an intersection of two events $\mathrm{A}$ and $\mathrm{B}$ is denoted by $\mathrm{P}(\mathrm{A}, \mathrm{B})$. Thus, $\mathrm{P}(\mathrm{OB}, \mathrm{Older})$ denotes the probability that a given sibling is both an older brother and an older sibling. Conditional probabilities are denoted as $\mathrm{P}(\mathrm{A} \mid \mathrm{B})$. For instance, $\mathrm{P}(\mathrm{OB} \mid \mathrm{Hom})$ denotes the probability that a sibling is an older brother, conditional on the event that the sibling is known to have been reported by a homosexual participant.

In order to distinguish the events which pertain to individual siblings from the number of times these events occur in a sample, we use the number sign (\#), which in this context should be read as "number of". Hence \#OB simply denotes the number of all reported older brothers in a sample. The subscripts Het or Hom are used to denote whether the total number of a given sibling type refers to that reported by homosexual or heterosexual participants. For instance the number of older brothers reported by homosexual participants is denoted by $\# \mathrm{OB}_{\mathrm{Hom}}$. 
We treat the terms "probability of an event" and "proportion of an event occurred" interchangeably, with the latter being an estimator of the former.

\subsection{The OBOR: Failure to quantify the effect of interest}

Blanchard $[5,8,16,19]$ warned that the proposed excess of older brothers due to the FBOE may go undetected by statistical tests if the mean number of all siblings reported by the group of homosexual men is appreciably smaller than the mean number of all siblings reported by the group of heterosexual men.1

To counter any potential mitigation of the relationship between homosexual orientation and the number of older brothers due to confounding effects of differences in family size between groups, Blanchard suggested that it is necessary to control for either the number of other siblings (\#Other) $[5,18,19]$ or the number of all siblings (\#All) [19] in a statistical model (i.e., test) comparing these two groups.2

Over the years, three new methods, designed to achieve this goal, have been developed, and increasingly are used in primary publications and meta-analyses on the FBOE. These are the already mentioned $O B O R$ [e.g., 5,13,24,25], the modified ratio of older brothers (MROB; [19]), and the modified proportion of older brothers (MPOB; [19]; e.g., [25]).

For now, we show that the $O B O R, M R O B$, and $M P O B$ are measures of the general excess of older siblings (i.e., brothers and sisters) among homosexual men, rather than measures of the more specific excess of older brothers only.

As the name implies, the $O B O R$ is an odds ratio, and it can thus be expressed in terms of conditional probabilities:

$$
\text { OBOR }=\frac{\mathrm{P}(\mathrm{OB} \mid \mathrm{Hom}) /[1-\mathrm{P}(\mathrm{OB} \mid \mathrm{Hom})]}{\mathrm{P}(\mathrm{OB} \mid \mathrm{Het}) /[1-\mathrm{P}(\mathrm{OB} \mid \mathrm{Het})]} .
$$

Now suppose we observe an $O B O R>1$, which, according to the interpretation of Blanchard et al. $[5,8,13,17,18]$, should be regarded as evidence for an excess of older brothers among homosexual men, and, by extension, for the FBOE.

For the $O B O R$ to become greater than unity, the numerator in Equation 1 must be greater than the denominator. Formally, this relationship can be stated as

$$
\text { OBOR }>1 \Leftrightarrow \mathrm{P}(\mathrm{OB} \mid \text { Hom })>\mathrm{P}(\mathrm{OB} \mid \text { Het })
$$

By conditioning on the event Older, the law of total probability (e.g., [26]) allows for the factorisation of both $\mathrm{P}(\mathrm{OB} \mid \mathrm{Hom})$ and $\mathrm{P}(\mathrm{OB} \mid \mathrm{Het})$, as follows:

$$
\begin{aligned}
& \mathrm{P}(\mathrm{OB} \mid \text { Hom })=\mathrm{P}(\mathrm{OB} \mid \text { Older,Hom }) \mathrm{P}(\text { Older } \mid \text { Hom }) \\
& +\mathrm{P}\left(\mathrm{OB} \mid \text { Older }^{\mathrm{c}}, \text { Hom }\right) \mathrm{P}\left(\text { Older }^{\mathrm{c}} \mid \mathrm{Hom}\right) \text { and, } \\
& \mathrm{P}(\mathrm{OB} \mid \text { Het })=\mathrm{P}(\mathrm{OB} \mid \text { Older }, \text { Het }) \mathrm{P}(\text { Older } \mid \text { Het }) \\
& +\mathrm{P}\left(\mathrm{OB} \mid \text { Older }^{\mathrm{c}}, \text { Het }\right) \mathrm{P}\left(\text { Older }^{\mathrm{c}} \mid \mathrm{Het}\right) \text {. }
\end{aligned}
$$

It is impossible for a sibling to be both, an older brother and a younger sibling (i.e., Older ${ }^{c}$ is impossible). Therefore, $\mathrm{P}\left(\mathrm{OB} \mid \mathrm{Older}^{c}, \mathrm{Hom}\right)$ and $\mathrm{P}\left(\mathrm{OB} \mid \mathrm{Older}^{c}, \mathrm{Het}\right)$ are both zero, reducing the above factorisations to

1 The term "undetected" refers to the lack of statistical significance of a size- $\alpha$-test of a respective effect size. Per common practice, $\alpha$ is chosen to be .05 and there seems to be some allowable margin of freedom or variation as to whether the alternative hypothesis should be one-sided or two-sided [15].

2 This necessity is claimed to have been demonstrated in [19], which upon closer examination reveals itself as being based on a circular argument and thus it is scientifically vacuous. Blanchard [19] assumed that a hand-picked set of real participant data contained evidence for an excess of older brothers in homosexual men and then concluded that the data showed evidence of an excess of older brothers among homosexual men, when using a transformation of predictor variables which supposedly controls for \# All or \#Other. These transformations are known as the Modified Ratio of Older Brothers (MROB) and the Modified Proportion of Older Brothers (MPOB; Table 2) and are discussed in detail below. 
$\mathrm{P}(\mathrm{OB} \mid$ Hom $) \quad=\mathrm{P}(\mathrm{OB} \mid$ Older,Hom $) \mathrm{P}($ Older $\mid$ Hom $)$, and

$\mathrm{P}(\mathrm{OB} \mid$ Het $) \quad=\mathrm{P}(\mathrm{OB} \mid$ Older,Het $) \mathrm{P}($ Older $\mid$ Het $)$.

One can think of $\mathrm{P}(\mathrm{OB} \mid$ Older,Hom $)$ and $\mathrm{P}(\mathrm{OB} \mid$ Older,Het $)$ as first restricting the sampling space from the set of all reported siblings to the subset which contains only older siblings and then computing the proportion of brothers within this subset for the homosexual and heterosexual group, respectively. In combining Equations 2 and 3 we obtain the following equivalent inequalities:

$$
\begin{aligned}
\text { OBOR } & >1 \\
\Leftrightarrow \mathrm{P}(\mathrm{OB} \mid \mathrm{Hom}) & >\mathrm{P}(\mathrm{OB} \mid \text { Het }) \\
\Leftrightarrow \mathrm{P}(\mathrm{OB} \mid \text { Hom, Older }) \mathrm{P}(\text { Older } \mid \text { Hom }) & >\mathrm{P}(\mathrm{OB} \mid \text { Het, Older }) \mathrm{P}(\text { Older } \mid \text { Het }) .
\end{aligned}
$$

Notice that Equation 4 (i.e., an $O B O R$ larger than unity) can hold even if $\mathrm{P}(\mathrm{OB} \mid \mathrm{Hom}$,Older $) \leq$ $\mathrm{P}(\mathrm{OB} \mid$ Het,Older). That is, one may observe that the proportion of older brothers in the subset of older siblings is smaller in the homosexual group, as opposed to the heterosexual group. However, the $O B O R$ taking into account the entire set of reported siblings- may still be greater than 1 , due to the proportion of older siblings being sufficiently greater in the homosexual group (i.e., $\mathrm{P}($ Older $\mid \mathrm{Hom})>\mathrm{P}($ Older $\mid$ Het $)$ ). This observation is incompatible with the FBOE, as the homosexual group must show a greater proportion of older brothers among older siblings. Otherwise there is no way that older brothers could be causally involved in the odds for homosexual orientation. The OBOR thus bears great resemblance to Simpson's paradox [27], in that not accounting (or in this case: insufficiently accounting) for a relevant confounder (here: Older) might wrongly lead to the opposite conclusion. Thus, the $O B O R$ at best provides a subpar answer to the more general, and thus irrelevant, question [28] of whether there is an excess of older siblings (not just brothers) among homosexual men.

It follows that -contrary to the claims in $[5,8,18]$ - for an $O B O R$ of 1 to even remotely be considered as adequately representing the theoretical null hypothesis of no FBOE, the probability (or proportion) of observing an older sibling must be identical in both groups. To see this, suppose that $\mathrm{P}(\mathrm{OB} \mid \mathrm{Older}, \mathrm{Het})$ and $\mathrm{P}(\mathrm{OB} \mid \mathrm{Older}, \mathrm{Hom})$ are equal to .515 , which is a widely agreed upon population estimate for the probability of being born male [29]. Further assume that the probabilities of observing an older sibling in the homosexual and heterosexual groups are equal to the median proportions of older siblings of $\mathrm{P}($ Older $\mid$ Hom $)=.58$ and $\mathrm{P}($ Older $\mid$ Het $)=.48$ reported across the 45 samples included in the three metaanalyses using the OBOR $[5,8,13]$. The OBOR under the null hypothesis is then given by

$$
O B O R_{\mathrm{H}_{0}}=\frac{(.515 \times .58) /(1-.515 \times .58)}{(.515 \times .48) /(1-.515 \times .48)}=1.30 .
$$

The fourth meta-analysis [5] reported an estimated random-effects mean $O B O R$ of $1.47,95 \% C I=[1.33$, 1.62] combining a total of 30 (31; see Part III) samples. For a distinct subset of 18 samples denoted "nonfeminine/ cisgender" men, Blanchard [5] reported a mean $O B O R$ of $1.27,95 \% C I=[1.20,1.35]$. Both of these estimates can be regarded as more or less compatible with the value of 1.30 derived in Equation 5 . In a commentary on Blanchard [5], Zietsch [30] cautioned about the available evidence on the FBOE, mainly due to the questionable and arbitrary inclusion criteria used in [5] leading to the exclusion of all probability samples known at that time.3

Blanchard replied by putting forth new evidence for the FBOE in a fifth meta-analysis [13], including probability samples only. The estimated mean OBOR over six such probability samples was $1.21,95 \% C I=$ $[1.13,1.30]$ and thus incompatible with the null-hypothesis of $O B O R=1$. However, in light of the above Equation (5), this estimated OBOR appears compatible with a model of an excess of older siblings, rather than a genuine excess of older brothers among homosexual men.

3 The term "probability sample" was defined by Zietsch [30] as "samples selected randomly with respect to the independent variable - sexual orientation in this case" (p.1). 


\subsection{Addressing the excess of older sisters}

It appears that Blanchard and colleagues were aware of the $O B O R$ s inability to distinguish between a genuine excess of older brothers and an excess of older siblings, as shortly after the fifth meta-analysis, the older sisters odds ratio (OSOR; see Table 2) was introduced [17]. Analogous the OBOR, the OSOR is simply the odds ratio of older sisters among all the siblings reported by homosexual and heterosexual men. Blanchard [17] reported that, for 29 out of the 36 samples included in the fourth and fifth metaanalyses, the $O B O R$ was numerically greater than the OSOR. However, this result reveals nothing about the size of the difference in the OBOR and the OSOR and might have led to a different conclusion if these differences had been properly weighted. In a similar vein, the seventh meta-analysis [8] considered both the $O B O R$ and the OSOR in a set of 24 samples and estimated a random-effects mean OBOR of $1.28,95 \%$ $C I=[1.22,1.35]$ for the entire set (which again is an estimate very compatible with the value derived in Equation 5). In addition, Blanchard et al. [8] reported estimated random-effects mean OSORs of 1.11, 95\% $\mathrm{CI}=[1.05,1.17]$ for what they denoted the "teleiophiles" subgroup and $1.15,95 \% \mathrm{CI}=[0.99,1.34]$ for the "pedophiles and hebephiles" subgroup. Blanchard et al. [8] describe pedophiles as men attracted predominantly to children before puberty, hebephiles as men predominantly attracted to children in puberty, and teleiophiles as individuals attracted to postpubertal, mature individuals.

The implication of the above is that -in order to ensure that estimated excesses of older brothers among homosexual men, when using the $O B O R$, is not an artefact of more general excesses of older siblings (i.e., older brothers and sisters)- one should interpret the $O B O R$ and the OSOR in tandem. However, having to interpret two separate, but correlated, statistics introduces an unnecessary degree of complexity.

Moreover, the apparent need to report both the $O B O R$ and the $O S O R$, as conveyed by [8], illustrates that it is the number (or equivalently, the proportion or probability) of older siblings that needs to be controlled for- not total family size or number of "other" siblings, as has repeatedly been asserted $[5,13,19]$.

This can be achieved by simply omitting the younger siblings from the denominator of the $O B O R$, and thus only the subset of older siblings is considered. The odds of observing an older brother in either group are now given by the ratio of older brothers to older sisters, and consequently the odds ratio $(O R)$ is given by

$$
\text { OR }=\frac{\# \mathrm{OB}_{\mathrm{Hom}} / \# \mathrm{OS}_{\mathrm{Hom}}}{\# \mathrm{OB}_{\mathrm{Het}} / \# \mathrm{OS}_{\mathrm{Het}}} .
$$

This $O R$ controls for the confounding effect of any differences in the probability of observing an older sibling in either group.

The numerator and denominator of Equation 6 may be conceived as the male-to-female sex ratio within the subset of older siblings among homosexual and heterosexual participants, respectively. Under the assumption of the FBOE and the implied excess of older brothers among older siblings among homosexual men, one would expect this ratio of sex ratios (i.e., an odds ratio) to be greater than unity.

We emphasize that the use of $O R$ s (the $O B O R$ and the $O R$ in Equation 6) implies a shift from the level of participants to the siblings reported by these participants and thus naturally introduces a hierarchical organization of the units of analysis (i.e., siblings are nested, or grouped, within participants). Thus, the statistical assumption of independent analysis units may be regarded as violated (see supplement S2 for further discussion on this topic).

\subsection{The modified ratio and proportion of older brothers}

The $M R O B$ (Table 2) can be regarded as an estimator of the odds of observing an older brother among all the siblings of the $i$ th participant, whereas the $M P O B$ (Table 2) can be regarded as an estimator of the probability of this event. Hence, given the one-to-one relation between odds and probabilities, the $M R O B$ and the $M P O B$ are related as follows:

$$
M R O B \approx \frac{M P O B}{1-M P O B}, \quad \text { and } \quad \mathrm{MPOB} \approx \frac{M R O B}{1+M R O B}
$$

Keeping in mind that we use proportions and probabilities interchangeably, the MPOB of the $i$ th participant is defined as the probability of observing an older brother within the sibship of the $i$ th participant, which can be written as 


$$
M P O B_{i} \approx \mathrm{P}(\mathrm{OB} \mid i)=\mathrm{P}(\mathrm{OB} \mid \text { Older }, i) \mathrm{P}(\text { Older } \mid i)
$$

Applying once more the law of total probability, it follows that the $M R O B$ for the $i$ th participant is given by:

$$
M R O B_{i} \approx \frac{\mathrm{P}(\mathrm{OB} \mid i)}{1-\mathrm{P}(\mathrm{OB} \mid i)}=\frac{\mathrm{P}(\mathrm{OB} \mid \text { Older, } i) \mathrm{P}(\text { Older } \mid i)}{1-\mathrm{P}(\mathrm{OB} \mid \text { Older }, i) \mathrm{P}(\mathrm{Older} \mid i)} .
$$

Blanchard [16,19] recommended either using the $M R O B$ or the $M P O B$ as predictors in a logistic regression model of participants' sexual orientation, as opposed to using the raw number of older brothers. Equations 7 and 8 suggest that it would not be difficult to come up with a scenario in which, on average, homosexual participants report just as many (or fewer) older brothers among their older siblings as heterosexual participants do, yet, due to more general group differences in the proportion of older siblings, the odds (or the probability) of observing an older brother still are greater for homosexual participants. Consequently any positive association between the probability of homosexual orientation and the odds (or probability) of observing an older brother among all of the siblings of a participant may be compatible with both scenarios, i.e., a genuine excess of older brothers but also with an excess of older brothers and sisters. Blanchard [19] defined two complementary indices for older sisters, the modified proportion of older sisters (MPOS) and the modified ratio of older sisters (MROS; see Table 2), and interpreted the presence of a statistically significant coefficient for the $M R O B(M P O B)$ and the simultaneous lack of a statistically significant coefficient for the MROS (MPOS) as evidence for the hypothesis that the number of older brothers, but not the number of older sisters, should be related to the probability of homosexual orientation in men. Declaring a significant difference between two effects (that of the $M R O B / M P O B$ and that of the MROS/MPOS) based on the observation that one effect statistically is significantly different from the null hypothesis, whereas the other is not, is a well-known fallacy in the application of statistical tests [31]. A pattern of statistically significant vs. statistically nonsignificant coefficients does not inform about whether the MROB s (MPOB s) effect can be taken to be greater than that of the MROS (MPOS).

As discussed next, the rationale for using the $M P O B$ and $M R O S$ seems to be based on a common misconception about ratios as "controlling" for the variable in the denominator [32], which was the stated purpose for introducing these measures into the FBOE literature in the first place [19].

\subsection{Ratios do not control for confounding variables}

As the sole predictor in a linear model, the $M R O B$ (and / or the MROS) is equivalent to including only the interaction term between the number of older brothers $(+0.33)$ and the reciprocal of the number of all other siblings (+1) into the model [33] (see corresponding equation in Table 2). However, the constituent variables of the interaction, (\#OB_i + 0.33) and 1/ (\#OS_i + \#YB_i + \#YS_i+1), are omitted from the model, which implies that the regression coefficients for \#OB+0.33 and $1 /(\# \mathrm{OS}+\# \mathrm{YB}+\# \mathrm{YS}+1)$ are both set to zero. That is, the statistical model contradicts the very theoretical model by assuming that neither the number of older brothers nor the number of other siblings have any effect on the odds of homosexual orientation. Similar considerations apply to the use of the MPOB and MPOS.

Ratios (often referred to as "indices") are ubiquitous in many areas in the social and behavioural sciences, and often their substantive interpretation appears straightforward and meaningful. The statistical analysis of ratios, however, is not at all straightforward (e.g., [32-34]). Most importantly, it is not the case that ratios control for the variable in the denominator (e.g., [33,34]). Instead, in order to control for the influence of an assumed confounding variable one could simply add the denominator constituent of a ratio as a predictor variable of its own to the statistical model.

For instance, a researcher might posit that the linear relationship between the log odds of homosexual orientation and the number of older brothers should be positive, but that the number of other siblings (\#Other; [19]) could attenuate the estimate of this relationship. It is evident that regressing sexual orientation (in units of log odds) on \#OB and \#Other would control for the confounding effect of \#Other. This regression model is a simple representation of the assumed theoretical model. In using the $M R O B$ $(M P O B)$ for modelling this relationship, the associated regression coefficient provides at best a biased 
estimate (positive or negative) of the regression coefficient for \#OB (the effect of interest); at worst, it introduces a spurious relationship between the predictor and outcome variables [33,34].

Nevertheless, in trying to interpret the regression coefficient for \# $\mathrm{OB}$, it becomes clear that controlling for the number of other siblings does not rule out the possibility of an older-sibling effect (i.e., older brothers and sisters). In a logistic regression (as recommended by Blanchard [19]), a positive regression coefficient for \#OB would indicate that -while holding the number of other siblings constant- the logit of the probability of homosexual orientation increases as a function of the number of older brothers. The problem with this model lies in holding \#Other constant, as there are numerous combinations of its constituent variables \#OS, \#YB, and \#YS, which all could add up to one and the same value for \#Other. That is, if the regression coefficient for \#OB were in fact driven by an older-sibling effect, participants who have more older brothers would also have more older sisters, whereas fewer younger brothers and younger sisters. In controlling for \#Other, this information is lost. By analogy, the variable \#All (the total number of siblings) is equally unfit for ruling out an older sibling effect, since numerous combinations of \#OB, \#OS, \#YB and \#YS would sum up to identical values of \#All.

There is thus no reasonable justification for controlling for \#Other and \# All [30]. The variable for which a confounding effect should be controlled for is the number of older siblings, \#Older (or, equivalently, the proportion of older siblings) [30,31,35].

To this end, Gelman and Stern [31] proposed the difference between \#OB and \#OS as one predictor and \#Older as a second predictor (in a logistic regression model). If solely the number of older brothers, but not the number of older sisters, were associated with homosexual orientation, then a positive regression coefficient for the difference between \#OB and \#OS should be observed (the number of older siblings). Alternatively, and in order to obtain an estimate of the increase in the odds of homosexual orientation, a model with the predictors \# $\mathrm{OB}$ and \#Older could be fitted to the data. This latter model, however, will introduce some collinearity, as the number of older brothers and the number of older siblings are correlated.

\subsection{Part I conclusions}

Our findings in Part I boil down to two overarching themes. First, the $O B O R$, the $M R O B$ and the $M P O B$ are all intended to control for the confounding effect of total family (or sibship) size in statistical analysis. Yet, it is the number of older siblings that must be controlled for instead, as has already been pointed out before $[30,31,35]$. Second, ratios do not control for the variable in the denominator, which is a common misconception surrounding the use of ratio variables [34]. Using basic probability calculus, the statistical clarification provided here has thus shown that ratios better should not be used in the way they are used in extant research on the FBOE, and that analyses need to control for the number of older siblings, but not the total family (or sibship) size.

\section{Part II: Assessing and comparing the performance of recommended and alternative measures using simulated data}

Next, we compare the performance of the statistical models recommended by Blanchard $[5,18,19]$ to models, which appropriately control for \#Older. To this end, we simulated data in $R$ [36] and assessed the frequency with which researchers would falsely find a genuine influence of older brothers on the odds of homosexual orientation, based on statistically significant regression coefficients from these models. We refer to this frequency as the false-positive rate. This definition of a false positive rate is conceptually different from what is understood as the false positive rate (or, the type-I-error rate) in the context of statistical hypothesis testing. The latter pertains solely to the false decisions about the parameter(s) of interest, not being included in the parameter space assumed under the null hypothesis, conditional on the specified model, and other statistical background assumptions [37]. In contrast, the use of the term falsepositive rate here encompasses the researchers' extra-statistical decisions of falsely declaring a substantive hypothesis (i.e., older brothers increase the odds of homosexual orientation) as corroborated based on the results of a hypothesis test of a parameter, within a falsely specified model. That is, the tested parameter (e.g., regression coefficient) does not meaningfully correspond to the substantive effect of interest, due to a falsely specified statistical model. 


\subsection{Methods}

We used the $R$ packages simstudy [38] (version 0.2.1), doRNG [39] (version 1.8.2), doParallel (version 1.0.15) [40], and foreach [41] (version 1.5.0) to generate random draws from a four-dimensional Poisson distribution with a specified correlation matrix, wherein each draw represented a simulated participant. The four dimensions served as the variables \#OB, \#OS, \#YB, and \#YS. The odds of homosexual orientation for participants with no older brothers were fixed at 0.02 [42]. Each older brother increased these odds by a factor of $(\theta+1)$ with theta $\in(-1, \infty)$. Thus, the simulated participants' odds of homosexual orientation were given by

$$
0.02(1+\theta)^{\# \mathrm{OB}} \text {. }
$$

We investigated the choices of $-0.33,0.33$ and 0 as possible values for $\theta$, with 0 representing no effect of older brothers on homosexual orientation at all, -0.33 a decrease in the odds of homosexual orientation per each older brother, and 0.33 a true increase in the odds of homosexual orientation per each older brother, which corresponds to the $33 \%$ increase reported in $[9,43]$.

The Poisson distribution was parameterised by a vector $\lambda$, the elements of which corresponded to the mean numbers of older brothers, older sisters, younger brothers and younger sisters, respectively. This vector of means (or rates) was determined by multiplying the mean number of all siblings, $\mu$, and the vector

$$
\left[\begin{array}{llll}
.515 \times \pi & (1-.515) \times \pi \quad .515 \times(1-\pi) & (1-.515) \times(1-\pi)
\end{array}\right]^{\mathrm{T}}
$$

, where .515 again represented the population estimate for the probability of male birth [29], and $\pi$ denoted the proportion of older siblings in a given sample.

For instance, for an average number of siblings of $\mu=3$ and equal probability that each sibling is an older or a younger sibling (i.e., $\pi=.5$ ), $\lambda$ would be given by

$$
\begin{aligned}
\lambda & =\left[\begin{array}{lllll}
.515 \times .5 & (1-.515) \times .5 & .515 \times(1-.5) & (1-.515) \times(1-.5)
\end{array}\right]^{T} \\
& =\left[\begin{array}{llll}
0.77 & 0.73 & 0.77 & 0.73
\end{array}\right]^{\mathrm{T}} .
\end{aligned}
$$

For the homosexual sample, the study employed three different values for $\pi$, namely $\pi=.5, \pi=.6$, and $\pi=.7$, while for the heterosexual sample, $\pi$ was fixed at .5; in other words, we simulated differences in the number (or proportion) of older siblings by increasing $\pi$ in the homosexual group.

The median of the mean numbers of all siblings for homosexual participants across the 45 samples in Blanchard [5], [13], and [8] was 2.45 .

In the equal condition of the simulation study, this value served as the mean number of all siblings (i.e., $\mu$ $=2.45$ ) for both the homosexual and heterosexual group. In the unequal condition, the mean number of siblings for each group were taken from the Mismatch 2 sample in [19], where the mean number of siblings in the homosexual group was $\mu=2.19$, and the mean number of siblings in the heterosexual group was $\mu=$ 3.31. Blanchard used the Mismatch 2 sample to demonstrate the inability of tests for mean differences and logistic regression to detect an older brother effect and promote the use of the $M R O B$ and the MPOB.

Due to the positive correlation between the number of older brothers and older sisters, homosexual males should also exhibit an excess of older sisters $[14,19,44]$. However, to our knowledge, no estimate of the magnitude of the correlation that would be required to bring about an artefactual older sister effect has ever been put forth. To address this issue, we incorporated our best guess of such an estimate, by imposing the correlation matrix of the four sibling types found in the male participants of Tran et al. [45] onto our simulated participants (Table 3; see https:/ / osf.io/3wnhu/ for data). This study consisted of 1,779 males, who provided complete information on the number of each sibling type (see the original publication for full description of the sample).

Thus, there were 18 possible combinations of $\theta, \mu$ and $\pi$. For each combination, we fitted 10 different models (see Table 4). 


\subsection{Models and sample-size considerations}

Table 4 lists the equations of the linear models we fitted to the simulated data. Model 1 in Table 4 predicted the logit of the probability of being homosexual, using the raw number of older brothers and older sisters as predictors. Blanchard [19] warned against using this model, stating that differences in the total number of siblings between homosexual and heterosexual participants (i.e., heterosexual participants having more siblings in general) may lead to the non-detection of a genuine older brother effect as conveyed by the regression coefficient of \#OB.

Models 2 and 3 used the MROB and MROS (Model 2) or the MPOB and MPOS (Model 3), respectively, instead of the raw numbers of older brothers and older sisters as predictors in a logistic regression model, as recommended in $[16,19]$.

The regression coefficients for the predictor $\mathrm{Hom}_{i}$ in Models 4 and 5 correspond to the natural $\log (\ln )$ of the OBOR (Model 4) and the OSOR (Model 5). Specifically, Model 4 predicted the logit probability of the $j$ th sibling reported by the $i$ th participant being an older brother (for Model 5: older sister), using only the homosexual orientation of the $i$ th participant as a predictor (abbreviated as Hom), with a value of 0 meaning no homosexual orientation and a value of 1 representing homosexual orientation. In Model 5, $\exp \left(\beta_{\mathrm{Hom}}\right)$ was not intended to capture the older-brother effect $(\theta)$ itself, but to assess a positive, but weaker, $O S O R$ (as compared to the $O B O R$ ), which should be observable in the presence of a genuine effect of older brothers on sexual orientation $[8,17]$.

Models 6 and 7 both included the raw number of older brothers as one predictor, but differed with respect to the second predictor, i.e., the variable whose confounding effect should be controlled for. Model 6 controlled for the number of all siblings, \#All, and Model 7 controlled for the number of siblings who are not older brothers (i.e., "other siblings", \#Other). These are the models actually implied by the claimed necessity of having to control for \#All and \#Other (as opposed to the $M P O B$ and the $M R O B$ ), respectively.

Models 8 through 10 controlled for the number of older siblings, \#Older [30,31,35]. Model 8 corresponds to the one proposed by [31] and included the difference between the number of older brothers and older sisters as the predictor of interest, while controlling for \#Older. A positive value for the regression coefficient of interest, $\beta_{\# \mathrm{OB}-\# \mathrm{OS}}$ would indicate a genuine excess of older brothers among homosexual men. Model 9 contains only the number of older brothers as the predictor of interest. While this may introduce some collinearity, the corresponding regression coefficient $\beta_{\# \mathrm{OB}}$ appears to be easier interpretable than $\beta_{\# \mathrm{OB}-\# \mathrm{OS}}$ in Model 8.

Finally, the regression coefficient for $\mathrm{Hom}_{i}$ in Model 10 is equivalent to the natural log of the odds ratio of older brothers to older sisters introduced in Equation 6, with the sampling frame being restricted to older siblings only. Model 10 predicted the logit of the probability of the $j$ th older sibling reported by the $i$ th participant being an older brother using the homosexual orientation (coded as 0 and 1 ) of the $i$ th participant.

Thus, just like Models 8 and 9, Model 10 controls for the number of older siblings, but is defined on the level of the reported siblings, rather than the level of participants.

For each model and each combination of $\mu, \pi$, and $\theta, 1000$ replications were carried out (i.e., 180,000 replications in total). Regression coefficients were deemed statistically significant for $p<.05$. Owing to the finite number of observations ( 1,000 replications per condition), the observed false-positive rates for the regression coefficients could differ from the underlying actual false-positive rate. We defined the margin of error for an acceptable true false-positive rate of .05 as follows: Falsely rejected null-hypotheses can be modelled as Bernoulli random variables with probability of success equal to .05 . Thus, the sum of 1,000 Bernoulli random variables (i.e., the number of falsely rejected null-hypotheses) is a Binomial random variable. Suppose, a test has a false-positive rate of .05 . The probability of observing 67 successes or less in 1000 trials (i.e., a false-positive rate of .067) is .99. Hence, we regarded an observed false-positive rate of greater than .05 , but smaller than .067 as compatible with a true false-positive rate of .05 . Sample sizes were determined by carrying out the simulation several times, increasing the sample size on each iteration until at least $80 \%$ of the regression coefficients for the predictor \#OB in Model 9 returned a $p<.05$ (two-tailed) given $\theta=0.33$. In other words, we increased the sample size until the coefficient of interest in Model 9 had a minimum approximate power of .8 , or more. Conditional on the alternative hypothesis (here: $\theta=.33$ ) 
being true, a test is equivalent to a Bernoulli random variable with probability of success equal to the test's power. Thus, the probability of observing 829 successes or fewer in 1,000 trials (i.e., a success rate of .829) is .99 . Thus, it is safe to conclude that for an observed rate of correctly rejected null hypotheses of 829 across 1,000 simulation replications, the hypothesis tests had a power of at least .8. A sample size of 700 participants per group seemed to be more than sufficient for this purpose (see results below). The rationale for tuning the sample size such that Model 9 had adequate power was that this model not only controlled for the number of older siblings but also provided a direct estimate for $\theta$ (i.e., the older brother effect in our simulation study) and was thus the most easily interpretable of the models.

\subsection{Results}

The results of the simulation study are displayed as error plots in Figure 1 (see online supplement for code, https: / / osf.io/3wnhu/). Each plot corresponds to one of the models in Table 4.

Model 1 (Figure 1) performed as described by Blanchard [19]. Given a difference in the mean number of all siblings taken from [19] (i.e., the $\mu$-unequal condition), a genuine excess of older brothers among homosexual men $(\theta=0.33)$ would have frequently been declared as a lack of older brothers, as indicated by the negative values of the estimated regression coefficients, $\widehat{\beta}_{\# \mathrm{OB}}$. In addition, the plot for Model 1 shows that these regression coefficients, which are estimates for $\theta$, were also affected by between-group differences in the proportion of older siblings. Thus, not only would Model 1 frequently lead to false conclusions about the direction of $\theta$, but also about its magnitude.

The plots for Models 2 and 3 show that interpreting the coefficient estimates of the $M R O B$ or the $M P O B$ in a logistic regression model (see Table 4) as corresponding to the effect of older brothers on homosexual orientation, would frequently lead to false conclusions about the presence of such an older brother effect. Furthermore, in following this line of inferring from these regression coefficients to the substantive effect of interest, researchers would frequently misidentify a negative older-brother effect (i.e., the odds of homosexual orientation decrease as a function of the number of older brothers due to $\theta=-0.33$ ) as a positive older brother-effect. The magnitudes of the $M R O B$ and $M P O B$ increased as the difference in the proportion of older siblings increased, as well.4

Similar considerations hold for the $O B O R$ (Model 4) and the OSOR (Model 5). Use of the OBOR (in combination with a test of $H_{0}: O B O R=1$ ) would frequently have led to the declaration of an older-brother effect, when in fact a greater proportion of older siblings $(\pi>.5)$ in homosexual men drove the increase in the $O B O R$. In some conditions, even genuine negative older-brother effects (i.e., $\theta=-0.33$ ) would have been declared as genuine positive older brother effects beyond the acceptable rate of false positives. Another noteworthy pattern in Models 4 and 5 is that under equal proportions of older siblings in both groups (i.e., $\pi=.5$ ), the mean $O S O R$ went in the opposite direction of the $O B O R$ slightly more often than would be expected under the acceptable false positive rate. This is interesting, as [8] reported a negative correlation between the $O B O R$ and the OSOR but did not further elaborate on it as it was not "statistically significant". Here, we observed that the $O B O R$ and $O S O R$ were negatively associated under conditions of equal proportions of older siblings between groups (which once again demonstrates the necessity of controlling for the number of older siblings).

The use of models, which actually control for \#All (Model 6) and \#Other (Model 7), would have also resulted in the false detection of an older-brother effect, way beyond the intended $5 \%$ rate. With respect to the qualitative assessment of the older-brother effect, Model 6 (i.e., controlling for \# All) behaved almost exactly as the $M R O B$ and $M P O B$ in Models 2 and 3. Controlling for \#Other (Model 7) would have

4 As shown in the supplementary materials (S4), when a genuine older-brother effect was present (i.e., $\theta=$ 0.33 ), the regression coefficients for the $M R O B$ and $M P O B$ were consistently greater in magnitude than the corresponding regression coefficients for the MROS and MPOS. This suggests that interpreting the two conjointly (for instance, by considering their difference) may yield decisions with a false-positive rate of $5 \%$. This however demonstrates the necessity of controlling for the number of older siblings, not the number of other (or all) siblings. 
frequently led to the false detection of a negative older brother effect (or to the nondetection of a genuine older-brother effect) under conditions of different mean numbers of all siblings between groups.

The results of Models 8 through 10 are straightforward. The corresponding plots in Figure 1 reveal that employing these models would have led to correct decisions about both the presence and the direction of an older-brother effect below the acceptable false positive rate of 5\%. Most importantly, these models contained meaningful (i.e., interpretable) regression coefficients, which remained unaffected across all combinations of $\mu$ and $\pi$.

\subsection{Summary of Parts I and II}

The results of Parts I and II demonstrate that currently recommended effect sizes, variable transformations, and their implementation in statistical models in the FBOE literature neither are needed, nor adequate for quantifying and distinguishing a genuine specific excess of older brothers from a more general excess of older siblings. Furthermore, models controlling for \#Other or \#All (Models 6 and 7) can be regarded as misspecifications and thus are prone to lead to false conclusions.

We also demonstrated the necessity of controlling for the number of older siblings, as group differences in the proportion of older siblings are bound to lead to the false detection of an excess of older brothers, when using the $O B O R, M R O B$, or $M P O B$. It is quite plausible to suppose such differences, as the difference in the median proportions of older siblings across the 45 homosexual and heterosexual samples in [5], [17], and [8] amounted to $10 \%$.

The $O B O R, M R O B$, and $M P O B$ fail to quantify the very thing, which they are advertised as quantifying. These measures and their recommended usage are not rigorous enough in exposing the proclaimed excess of older brothers to conditions under which observations could indicate the absence of such an excess.

Given that the most extensive meta-analyses to date (totalling 45 samples of homo- and heterosexual; $[5,8,13])$ all employed the $O B O R$, it follows that these meta-analyses actually provided no evidence for the FBOE and its associated claims. Our findings, of course, do not preclude the possibility that there may nevertheless be a meaningful excess of older brothers in homosexual men as predicted by the FBOE. This is one of the reasons for why in Part III we provide a set of new meta-analyses, using more adequate measures, along with an advanced meta-analytic framework.

\section{Part III: Multiverse (Specification-Curve) Meta-Analysis of the Literature}

The dismissal of the $O B O R$ as ill-conceived invalidates most of the meta-analytic evidence for an excess of older brothers among homosexual men and consequently the FBOE and its associated claims. Thus reanalysing [5], [13], and [8] using a more appropriate effect-size metric, appeared to be a necessity in order to fill the evidential void, once occupied by these meta-analyses. Moreover, the very existence of seven non-cumulative meta-analyses -each different with respect to the subset of available samples included (see Table 1) and the method used for amalgamating the evidence from these samples- demonstrates the mostly unacknowledged issue of researcher degrees of freedom [46] in specifying a single meta-analysis (see supplement S4 for a discussion of researcher degrees of freedom in primary analyses on the FBOE). In Part III, we therefore set out to address this issue of researcher degrees of freedom in meta-analyses by extending our re-analyses of [5], [13], and [8] to include alternatively specified meta-analyses over the same subsets of samples. Furthermore, and for the first time, we report the results of meta-analyses encompassing all extant male and female samples, thereby comparing the excess of older brothers among homosexual men to that among homosexual women, the difference of this being crucial to the verisimilitude of the MIH. Finally, we offer a glimpse at the "garden of forking paths" [47] of conceivable alternative meta-analyses for both male and female samples using the framework of specification-curve and multiverse meta-analysis [21-23]. To this end, we identified a number of necessary steps in specifying a single meta-analysis, where researcher degrees of freedom may possibly affect the outcome of any particular meta-analysis.

\subsection{Methods}

\subsubsection{Literature search and description of available samples}

We ascertained that the fourth, fifth, and seventh of the meta-analyses $[5,8,13]$ comprised 45 unique, nonoverlapping samples of homosexual and heterosexual male participants originating from 35 studies. This 
count deviates from the count of 44 samples reported by $[5,8,13]$ and can be traced back to Blanchard [5], who merged the two unrelated samples in Blanchard et al. [43] into a single sample. We could not see how this step could be precisely justified, and thus treated these two independent samples in [43] as separate.

We were able to retrieve all but one of the publications of the primary studies in [5], [13], and [8]. The study by Krupp was cited as an unpublished manuscript in [8]. The mean and / or total numbers of older brothers and older sisters, and the corresponding standard deviation were extracted, if these were reported or could be determined through reported statistics. Twenty-one of these primary publications did not contain enough information to deduce the mean number of older brothers and older sisters or the corresponding standard deviations of these. Thus, we drew on the summary table in the Appendix of Blanchard [5] and Table 1 of [8] to obtain the mean number of older brothers and older sisters for the following study samples: Blanchard and Sheridan [48], Blanchard and Zucker [49], Zucker and Blanchard [50], Blanchard et al. [51], Blanchard and Bogaert [1,2], Bogaert et al. [52], Blanchard and Bogaert [53] (all three samples), Ellis and Blanchard [54], Blanchard et al. [55] (samples named "Bogaert other", "Bogaert non-biological families" and "Blanchard"), Blanchard [56] (all three samples) and Krupp [8] (both samples). The mean numbers of older brothers and older sisters of three additional samples were obtained from Table 1 in Blanchard [13]. These samples were originally referenced in Bogaert [57] and Zietsch et al. [58] (two samples: "female co-twins" and "male co-twins"). The mean number of older brothers and older sisters and their standard deviations for the homosexual and heterosexual male samples in Frisch and Hviid [35] were obtained from Blanchard and VanderLaan [15]. We retrieved 19 additional samples through database searches, cited reference searches, and citation alerts (all of these based on Google Scholar). One of these samples was retrieved from [45] and had previously been analysed in a different context (the data are provided online, https: / / osf.io/3wnhu/).

The sole inclusion criterion used to obtain additional samples was that the number of older brothers (average or total) and older sisters among homosexual and heterosexual male participants had to be available, as the number of younger brothers are irrelevant to the estimation of any excess of older brothers among homosexual men. Furthermore, we also extracted the mean or total numbers of younger brothers and sisters, if these were reported in order to compare the magnitude of the $O B O R$ to the magnitude of the $O R$ for older brothers vs. older sisters (Equation 6 and Model 10 in Table 4).

Seventeen samples also reported sibling data on homosexual and heterosexual women; these data were also extracted (see Table 6). In order to obtain the mean number of each sibling type for the female samples in Frisch and Hviid [35], we used the same approach as Blanchard and VanderLaan [15] for obtaining the mean number of each sibling type for the male participants: Table 4 of Frisch and Hviid contained the number of participants who had $0,1,2$, or 3 or more siblings in each category (older brothers, older sisters, younger brothers and younger sisters). We multiplied the numbers of participants in each category by the respective numbers of older brothers, older sisters, younger brothers, and younger sisters, and then divided the resulting sums by the total numbers of participants. Theoretically, this may lead to a negative bias in the estimate of the mean number of each sibling type. However, we contend that this bias is practically negligible, since having three or more siblings of the same sex and sibship position may be regarded as an extremely rare event (for instance, only nine out of the 1,573 homosexual women in Frisch and Hviid [35] reported to have three or more older brothers).

While the standard deviation was not needed for the effect-size metric we employed for meta-analysis (i.e., the $\ln O R$ in Equation 6, see next subsection), we nevertheless decided to report these in Tables 5 and 6 below, in order to highlight the pattern of non-reporting of basic summary statistics in this research field. Of note, it is impossible, to deduce the standard deviations for a total of 28 of the 64 samples in Table 5, simply due to insufficient reporting, although the respective analyses in these primary studies were carried out on the participant level and thus most likely required standard errors to obtain a test statistic.

Most of the available samples were subjected to a binary classification scheme with respect to participants' sexual orientation. In addition, some samples contained sibship data of participants, whose sexual orientation was classified as bisexual $[24,25,45,59,60]$ or asexual [60,61], while in other samples bisexual participants were simply merged with the homosexual group [2,52,55,57,62-64]. 
Since the FBOE specifically states that the number of older brothers should increase the odds of homosexual orientation [13], the bisexual and asexual groups were excluded from Tables 5 and 6, whenever possible (In aside, we note that this decision is another obvious researcher degree of freedom).

Homosexual and heterosexual were not the only labels assigned to the two categories in the binary classification schemes used throughout the available samples. Other binary labels were heterosexual vs. nonheterosexual [58,61,65,66], homosexual vs. nonhomosexual [43,48,60,67], and androphilic vs. gynephilic [25,68-71]. In addition, two studies did not assess sexual orientation at all, but rather compared the sibship compositions of male-assigned individuals diagnosed with gender dysphoria (GDY) to heterosexual controls [43,72]. Blanchard [5] did not provide a justification as to why these samples should be included in his meta-analysis, 5 but given that [13] classified these participants as homosexual, it appears as if GDY could be regarded as a proxy for homosexual orientation. These varying classification schemes constitute yet another unchecked researcher degree of freedom in the process of devising an analysis plan.

In line with the fourth, fifth, and seventh meta-analysis $[5,8,13]$, the groups from these primary samples were categorized as either homosexual or heterosexual in Tables 5 and 6. Groups labelled as heterosexual, nonhomosexual, or gynephilic were assigned to the heterosexual category, whereas groups labelled as homosexual, nonheterosexual and androphilic were assigned to the homosexual category. For the two samples without assessment of sexual orientation [43,72], GDY participants were assigned to the homosexual group, whereas the controls to the heterosexual group (as in [5]). Tables 5 and 6 display only the mean numbers and standard deviations of older brothers and older sisters. The corresponding statistics for younger brothers and younger sisters are derivable from the data provided (https:/ / osf.io/3wnhu/).

\subsubsection{Data analysis}

In choosing an effect size for meta-analysis, we followed the approach of Blanchard et al., in modelling reported siblings as independent observations $[5,13,16]$. Note that no further information on the reported siblings was available than the number of times each sibling type occurred in a sample.

We quantified the excess of older brothers in the homosexual group through the natural log of the odds ratio (lnOR) of older brothers vs. older sisters as described in Equation 6 above and Model 10 in Table 4. As shown in Parts I and II, this lnOR controls for the number (or the proportion) of older siblings and remains unaffected by group differences in the total number of siblings. In order to compute this $\ln O R$, the total numbers of older brothers and sisters of the homosexual and heterosexual participants are needed, instead of the mean numbers listed in Tables 5 and 6 . These totals are easily obtained by multiplying the Tables 5 and 6 mean values with the corresponding sample size (column $N$ ) and rounding to the nearest integer. Alternatively, one could use the difference in the proportion of older brothers among older siblings between homosexual and heterosexual men and women as an effect size. Corresponding metaanalyses using the difference in proportions as an effect size can be found in the supplementary materials (S5).

We fitted both fixed-effect and random-effects models to five specific study sets comprising the 31 male samples in [5], the six male probability samples in [13], the 24 male samples in [8], the entire set of 64 male samples, and the 17 female samples. For all random-effects models, the between-sample variance $\tau^{2}$ was estimated using the restricted maximum-likelihood (REML) estimator implemented in the $R$ package metafor [73].

To further assess the extent to which the effect might differ between men and women, we also computed a combined meta-analysis over the entire set of 81 (male and female) samples, with participant sex (male vs. female) in the sample serving as a covariate. Because each female sample was embedded in a study alongside a male sample (there are no all-female studies in this literature to our knowledge), there may be some degree of dependency between effect-size estimates from the same study. This study-level dependency can be modelled using a three-level-meta-analysis [74], which requires the estimation of a second, study-level variance component $\left(\tau_{2}^{2}\right)$ in addition to the first, sample-level variance component $\left(\tau_{1}^{2}\right)$.

5 The use of proxy variables for classifying sexual orientation was listed as one of the five exclusion criteria in Blanchard [5]. 


\subsubsection{Specification-curve and multiverse meta-analysis}

Invariably, the results of any meta-analysis are subject to a sequence of researcher-dependent decisions pertaining to the inclusion of studies, the assumed meta-analytic model and the estimation of its parameters [21]. For instance, [5] defined the proximal assessment of sexual orientation via same-sex marriage as sufficiently invalid to justify the exclusion of one of the largest samples [35] available. In contrast, samples comprised of individuals whose sexual orientation was classified using phallometric testing [55,75], categorizing children as young as two years of age as "prehomosexual" [51,76], based on the "femininity" rating of their behaviours and expert ratings of criminal or clinical records $[48,52,53]$, were considered worthy of inclusion. Other researchers may disagree and argue that same-sex marriage constitutes a less error-prone indicator of homosexual orientation than changes in penile blood volume upon exposure to certain stimuli (as is the case in phallometric testing) and that the latter study -not the former one- should be noneligible for meta-analysis. Consequently, other researchers could opt to analyse a different set of samples than Blanchard [5] and therefore obtain different results. The perceived validity differing approaches for classifying sexual orientation is just one out of various crucial steps in the sequence of researchers decisions which lead to a particular meta-analysis.

With regards to the decision of which meta-analytic model should be fitted to the data, Blanchard and colleagues [5], [17], and [8], solely reported the results of random-effects meta-analyses. Researchers may disagree with Blanchard et al. and argue in favour of a fixed-effect model. Furthermore, fixed-effect and random-effects models provide answers to different inferential questions [77,78]. By fitting a randomeffects model, Blanchard and colleagues $[5,8,17]$ implicitly defined the mean and variance of an assumed distribution of population effects as the inferential goal of their statistical analysis. However, their interpretation of the size and consistency (or "reliability" [5]) of the OBOR solely relied on the estimated mean of this distribution, thus neglecting a substantial amount of available information on this distribution's variability [79]. More precisely, when the assumed data-generating process is estimated to having produced highly variable effects across populations, the mean of this distribution alone conveys little information about "the" effect (here: "the" $O B O R$ ), as in such a scenario it does not make sense to speak of a single effect at all, let alone to infer that it is consistent.

There are many more such decision steps or researcher degrees of freedom in specifying a meta-analysis, thus demanding researchers to choose one out of several possible alternatives, and for each combination of such alternatives, a different meta-analytic model is specified. Thus, for each reported meta-analysis, there is a sizeable collection of unreported, alternatively specified, meta-analyses, which may lead to considerably different conclusions.

Instances of disagreement among researchers about how a given set of data should be analysed are not confined to the realm of meta-analysis. The numerous degrees of freedom researchers have at their hands in primary data analysis are well documented $[22,23,46,80]$. Pointing out the issue of researcher degrees of freedom in a given analysis may occasionally be taken up as an accusation of questionable research practices (e.g., " $p$-hacking", "fishing for statistical significance") [47]. Yet, even in the absence of questionable research practices, researchers may still disagree considerably with respect to how the given data should be analysed, and thus how the research question should be optimally answered (e.g., [80]).

Given the obvious structural similarities between primary data analysis and meta-analysis (effect sizes correspond to individual participant data), specification-curve and multiverse meta-analysis [21] builds on two almost identical approaches for tackling the issue of researcher degrees of freedom, taken from the realm of primary data analysis, namely specification-curve analysis [22] and multiverse analysis [23]. These approaches identify all reasonably specified analysis plans and report all of the corresponding results. The set of results obtained then serves as the foundation for an "extra-data-analytic" step of inferring the state of the substantive research question. For the identification of reasonable analysis plans, [22] and [21] suggested to survey potential decision steps in the process of devising an analysis and to determine all possible combinations of the alternatives offered at each decision step. All nonredundant data-analytic specifications retrieved in this manner are then considered as reasonable specifications. We follow the terminology introduced by [21] and refer to the decision steps in specifying a meta-analysis as Which and How factors. The Which factors refer to decisions leading up to the set of samples (or studies) 
included in a meta-analysis (i.e., which primary studies are included in a meta-analysis) and are mostly determined by features of the samples themselves. The How factors subsume the decisions leading up to the technical implementation of a meta-analysis over a given set of samples, such as the selected metaanalytic model and the effect-size metric (i.e., how the primary studies are meta-analysed). Table 7 lists the ten Which factors, which we derived by scrutinizing the inclusion criteria of prior related meta-analyses $[5,13,15$, and 8$]$, from one critical commentary by [30], and from our own concerns.

(1) The factor In Previous Meta-analysis indicated whether a sample had been included in any of the seven previous meta-analyses $[5,7,13-15,18]$. Given that all of these were conducted by the same group of researchers (spearheaded by Blanchard), some samples may well have been judged more favourable for meta-analytic inclusion (possibly based on prior convictions that an excess of older brothers in homosexual men is real).

(2) The factor Labindicated whether Blanchard, Bogaert, Zucker or VanderLaan were listed as a coauthors of the publications the samples appeared in. These researchers frequently collaborated on FBOE publications.

(3) The factor Archives indicated whether a study was published in the journal Archives of Sexual Behavior. Thirty-two out of the 64 male samples appeared in articles published in this journal. As of late 2020, Zucker served as Editor, VanderLaan as Managing Editor, and Blanchard and Bogaert as Editorial Board members of this journal (https:/ / www.springer.com/journal/10508/editors).

(4) The factor Feminine indicated whether a given sample had been declared a "feminine" sample by [5], wherein it was theorized that the excess of older brothers is stronger in "feminine" samples, as opposed to "non-feminine/ cisgender" samples.

(5) The factor $G D Y$ indicated whether samples comprised individuals diagnosed with GDY. With exception of Vasey and VanderLaan [71] and the replication sample in VanderLaan and Vasey [70], all "feminine" samples comprised individuals diagnosed with GDY. It might be argued that such samples should be excluded from meta-analysis, due to generalisability concerns of their results.

(6) The factor Clinical indicated whether participants were recruited in a clinical setting. This issue was pointed out by [30], who questioned the representativeness of such samples.

(7) The factor Classification distinguished between four different methods for classifying sexual orientation, as used in the FBOE literature. We assigned each sample's classification scheme to one of the three broad categories (i.e., factor levels), which reflect different levels of reliability of classification. The factor-level Single-Item referred to studies which determined sexual orientation via a single piece of information (such as asking participants which category they would ascribe themselves to, or whether participants were in a same-sex relationship [35]), as opposed to a summary score of multiple items or expert ratings. The factor level Various subsumed samples, for which sexual orientation had been determined by computing summary scores over multiple items (such as the Kinsey Scale), or which combined participants whose sexual orientation had been assessed via a mixture of methods, such as the "Blanchard"-subsample in [55]. The factor level Indirect was assigned to samples wherein participants were categorized by either employing behavioural measures (e.g., phallometric testing, feminine behaviours in children as predictors of homosexual orientation), criminal offence records, or expert ratings to determine sexual orientation. Also, two studies [2,72] did not classify sexual orientation per se (but used GDY diagnosis as a proxy). These studies are indicated by the factor level none.

(8) The factor Probability indicated whether a sample was declared a "probability sample", i.e., samples comprised of participants who were randomly selected, with respect to sexual orientation [30], or samples that had been obtained through some kind of random sampling procedure [25,81]. All other samples were regarded as non-probabilistic convenience samples.

(9) The factor Stopping Rule indicated whether the authors of a primary study or Blanchard [5] assumed the presence of a stopping rule, such as one-child policies (e.g., [64]), or parents ceasing to reproduce after having at least one male and one female offspring [62]. Blanchard [13] noted that the proposed excess of older brothers may go undetected in the presence of a stopping rule.

(10) Finally, the factor Pedo-/Hebephiles indicated whether a sample comprised individuals who were classified as paedophile or hebephile by [8] (this factor was investigated as a potential moderator in [8]).

We considered only a single factor in terms of how to analyse these subsets of samples from the corpus of primary studies (i.e., the How factor), namely, fitting a random effects model (as in $[5,8,13]$ ) or a fixedeffect model.

Counting all the factor-level combinations (considering also their union, where applicable) of the ten Which factors and the single How factor resulted in a total of 3 (In Previous Meta-Analysis) $\times 3$ (Lab) $\times 3$ (Archives) x 3 (Feminine) $\times 3$ (GDY) $\times 3$ (Clinical) $\times 5$ (Classification) x 3 (Probability) $\times 3$ (Stopping Rule) $\times$ 3 (Pedo-/Hebephiles) $\times 2$ (fixed effect vs. random effects) $=196,830$ possible meta-analytic specifications for the 64 male samples. This set of specifications comprised 1,638 unique, non-redundant combinations of at least two from the 64 male samples in Table 5 (i.e., the set of reasonable specifications; see analysis code 
provided online; https: / osf.io/3wnhu/). Owing to the lack of previous meta-analyses of an older brother effect in female samples, the factor In Previous Meta-Analysis was not applicable when determining the subset of possible meta-analyses for female samples, as was the case for the factors Feminine and Pedo/Hebephile. For the remaining applicable factors, there were 3 (Lab) $\times 3$ (Archives) $\times 3$ (GDY) 33 (Clinical) x 5 (Classification) x 3 (Probability) x 3 (Stopping Rule) $\times 2$ (fixed effect vs. random effects) $=7,290$ possible meta-analytic specifications. Of these, 212 turned out to be unique and non-redundant specifications comprising at least two from the 17 available female samples.

All analyses were carried out in $R$ (version 4.0.3) [36]. Meta-analyses were computed using the metafor package [73], graphical displays of results were generated using metaviz [82], and ggplot2 [83] (see online material for code; https:/ / osf.io/3wnhu/).

We follow recommendations [84] of stating how the reported interval estimates were obtained. Confidence intervals for summary $\ln O R$ s are based on the normal approximation to the sampling distribution of the maximum likelihood estimators (i.e., the lnORs; these intervals being the default option in metafor [73]). Confidence intervals for the between-sample standard deviation, $\tau$, are based on the profile likelihood of $\tau^{2}$ [85], which is the default when fitting meta-analyses using the rma.mv() function in the metafor package [73]. Prediction intervals were obtained as described in [79] (this is also the default option in metafor).

Further, in reporting the results of the meta-analytic specification-curves for male and female samples, we also make use of the $S$-value [86]), a mapping of the $p$-value onto an additive scale without an upper bound, given by $S=l d(1 / p)$, where $l d$ denotes the base 2 logarithm. Like the $p$-value, the $S$-value indicates the degree of compatibility between the observed data and the statistical null model, which not only comprises the null hypothesis but also a host of statistical background assumptions, pertaining to the assumed data generating distribution, the absence of selection bias, and the sufficiently correct specification of the model, among other possible assumptions [86,87]. The $S$-value is interpretable as bits of information against the null model. For instance, a (just-significant) $p$-value of .05 corresponds to an $S$ value of 4.32 , i.e., conveys 4.32 bits of information against the null model. The unit of bits can further be interpreted as a surprisal value for observing a given $S$-value. An $S$-value of 4.32 is as surprising as observing four heads out of four tosses of a fair coin (which is not much surprising and certainly would not already justify the conclusion that this coin is unfair).

\subsection{Results}

\subsubsection{Meta-analyses of the five specific study sets}

Table 8 provides a summary of the meta-analyses over the five specific subsets of male and female samples. There was little disagreement between the estimated summary $\ln O R$ s across all four specific male study sets and fixed- versus random-effects models. Depending on which specific study set is interpreted, the odds for observing an older brother among the set of all older siblings reported by homosexual participants (male or female) were between 3\% (fixed-effect model for the six probability samples included in [13]) and 17\% (random-effects model for the 31 samples included in [5]) greater than those same odds for the heterosexual participants. However, the 95\% CIs suggest that these estimates were compatible with a 7\% decrease (i.e., lower bound of the $95 \% \mathrm{CI}$ of the fixed-effect summary estimate for the 6 probability samples included in [5]) as well as a 35\% increase (i.e., upper bound of the $95 \% \mathrm{CI}$ of the random-effects summary estimate for the 6 probability samples in [5]) for these odds. While the precision of these summary $\ln O R$ s varied considerably across the specific study sets and meta-analytic models (the shortest 95\% CI spanned $0.06 \ln O R \mathrm{~s}$, the widest 95\% CI spanned $0.36 \ln O R \mathrm{~s})$, all of the point estimates were directionally consistent with an excess of older brothers among the older siblings of homosexual men. This excess was not specific to the male samples, however, as the summary $\ln O R$ s for the female samples were also consistent with an excess of older brothers in homosexual women, with an estimated $7 \%$ greater odds of observing an older brother among the older siblings of homosexual vs. heterosexual women.

Comparing the summary estimate of all 64 male to that of all 17 female samples, we observed a difference of $-0.004,95 \% \mathrm{CI}=[-0.061,0.053]$, in the fixed-effect model, and a difference of $0.07,95 \% \mathrm{CI}=[-0.08,0.22]$, 
in the random-effects model. The three-level meta-analysis returned results almost identical to those of the random-effects model, with a between-group difference of $0.06,95 \% \mathrm{CI}=[-0.08,0.20], \hat{\tau}_{1}=0.12,95 \% \mathrm{CI}=$ $[0.00,0.22], \hat{\tau}_{2}=0.11,95 \% \mathrm{CI}=[0.00,0.22]$. These results suggest that conditional on the assumed statistical model(s), the available data do not convey enough information to confidently declare the respective excess of older brothers in homosexual men as different from an excess of older brothers in homosexual women. To put things in perspective: this inconclusive finding is based on the data of 30,000 homosexual individuals (17,134 older brothers and 15286 older sisters reported). Moreover, it calls into question previous confident claims about the excess of older brothers in homosexual individuals being specific to males [e.g., 10,52].

To facilitate the comparison of the magnitude of the $O R$ for older brothers versus older sisters to that of the $O B O R$, Table 8 also lists the results of the meta-analyses using the $\ln O B O R$ as an effect size. It is evident that if the $O B O R$ is falsely interpreted as quantifying the effect of interest, one would exaggerate the magnitude of the effect in male samples considerably.

With regards to effect-size heterogeneity, the $\hat{\tau} \mathrm{s}$ for the random-effects models were all of the same magnitude as the corresponding summary $\ln O R s$ themselves. Population effects thus appeared highly variable across samples. The $95 \%$ prediction intervals (PI) suggest a wide range of effects, which are consistent with both an excess and a lack of older brothers among homosexual men and women.

Blanchard [5] (fourth meta-analysis) found that some of the heterogeneity of the OBOR could be attributed to two distinct subgroups of samples, namely "feminine" samples and "non-feminine/ cisgender" samples. The re-analysis of [5] using the $\ln O R$ returned fixed-effect summary estimates of $0.09,95 \% \mathrm{CI}=[0.03,0.16]$ for the "non-feminine/ cisgender" subgroup of samples and $0.29,95 \% \mathrm{CI}=[0.17,0.40]$ for the "feminine" group. In the random-effects model, these estimates were nearly identical, $\ln O R=0.09,95 \% \mathrm{CI}=[0.03$, $0.17]$ and $\ln O R=0.29,95 \% \mathrm{CI}=[0.17,0.41]$. Across all 47 samples which were not denoted "feminine" by [5], we obtained fixed-effect estimates of $\ln O R=0.05,95 \% \mathrm{CI}=[0.01,0.08]$ and random-effects estimates of $\ln O R=0.08,95 \% \mathrm{CI}=[0.02,0.13]$. This subgroup analysis suggests a sizeable difference in the effect between "feminine" and "non-feminine/cisgender" individuals

Figure 2 displays a raindrop forest plots [82,88] accompanying the random-effects meta-analysis of all male sample $\ln O R$ effect sizes (the respective plot of the fixed-effect meta-analysis is provided in supplement S6). Therein, the observed effect size estimates are arranged (from top to bottom) in an ascending order of their meta-analytic weight. Two patterns are visible in Figure 2. First, only seven out of $64(11 \%)$ confidence intervals' lower bounds were greater than zero. That is, only seven samples returned a statistically significant effect in the right direction when using the (adequate) lnOR effect size metric. This contrasts the "consistent" and "reliable" (e.g., $[7,15])$ excess of older brothers among homosexual men previously reported in all these primary studies.

Second, with increasing study weight, the effect estimates seemed to approach zero. This is indicative of small-study effects (i.e., smaller, less precise, studies report larger effects than larger, more precise, studies). The funnel plot in supplement S6 further illustrates this observation. Assuming the absence of publication bias, one would expect the observed effect sizes to be distributed symmetrically around the summary effect estimate [89]. This was visually not the case in Figure S4 and the observed effect sizes compatibility with a model assuming symmetry of effect sizes, as was indicated by Egger's regression ([90]): Regressing the observed effect sizes on their standard errors, the regression coefficient for the standard error was $0.70,95 \% \mathrm{CI}=[0.35,1.05]$, in the fixed-effect model, and $0.80,95 \% \mathrm{CI}=[0.28,1.31]$, in the random-effects model. This indicates that (when rescaling these coefficients by dividing them by 10), on average, two effect sizes differing by 0.1 standard errors are expected to differ by 0.07 lnORs $(0.08 \mathrm{lnORs}$, respectively). This difference is not only sizeable in comparison to the fixed-effect summary estimate of 0.07 , but also in comparison to all the other summary estimates listed in Table 8. Sample size contributes less to study weight in the random-effects model than in the fixed-effect model. Hence, small-study effects (if they go into the direction of the desired effect, as was the case in the present data) may explain the larger summary estimates in the random-effects models, compared to those of the fixed-effect models in Table 8.

Figure 3 presents an Albatross plot [91] of the $p$ values of all individual male sample lnOR effect sizes. Text-book examples of publication bias analysis mostly assume a simple selection model, wherein the chances of a study being published is a function of the statistical significance associated with the effect of 
interest. The Albatross plot visually highlights various p-value regions, draws contours for the range of effect sizes, which may have led to the observed p-values, and may help in the identification of effect-size heterogeneity. Even though any $p$ value can be used for the Albatross plot (allowing for the aggregation of vastly different effect metrics and study designs), we used the $p$ values of the lnOR effect sizes here. The multiplicity of outcomes and analyses in primary studies obviated the selection of unambiguously "representative" $p$ values for each study. Hence, the Albatross plot, as well as the foregoing effect size distribution analysis, only approximated the evidence originally presented in the available primary studies. Both analyses had to be based on effect sizes, which had not been directly reported in the primary studies. The contours in the Albatross plot indicated considerable heterogeneity in the data, with only a small number of studies suggesting a large effect $(\mathrm{lnOR}=0.69)$, but a clear majority of studies (around 54 $[84 \%])$ a considerably smaller effect $(\operatorname{lnOR} \leq 0.26)$. Twenty-two studies $(34 \%)$ indicated $\operatorname{lnOR}<0.12$. Larger studies reported on average smaller effects. Moreover, the plot highlighted a number of studies (around 8 [13\%]), all of which suggested $\operatorname{lnOR} \geq 0.12$, that closely missed significance (around $05 .<\mathrm{p}<.1$ ) or were just barely significant $(01 .<\mathrm{p}<.05)$.

\subsubsection{Specification-curve and multiverse meta-analyses}

To demonstrate, which other sample-specific factors could potentially influence the estimates of the metaanalytic means and thus the qualitative conclusion about the presence of an excess of older brothers in homosexual men, we now consider the results of the specification curve meta-analyses described above as well as in [21].

The results of the 1,628 and 212 non-redundant meta-analytic specifications for the set of male and female samples, are summarized in Figure 4. From top to bottom, the panels encode each specification's estimated summary effect and 95\% confidence interval, number of samples included (density plot), and combination of factor levels (tile plot). The point estimates are arranged in ascending order and are connected by a black solid line - the specification curve. In addition, each specification is color-coded, using hues of six distinct colours (red, orange, yellow, green, blue, violet) on the spectrum from red to violet. A specification's colour and hue are indicative of the number of samples included in the meta-analysis, with the number of samples increasing in the following order: red, orange, yellow, green, blue, violet.

In terms of magnitude, the estimated mean $\ln O R s$ of the various subsets of male samples ranged from $0.29(O R=0.74)$ to $0.93(O R=2.53)$, with an interquartile range of 0.05 to $0.14(O R=1.05$ to 1.15$)$. In total, $96 \%$ of the estimated means were greater than 0 , and $47.4 \%$ of these had $95 \%$ CIs that did not include 0 (i.e., estimated means greater than 0 , with $p<.05$ (two-tailed)).

The overall pattern of the specification curve seems to indicate that the specifications with many samples and/or narrow confidence intervals were closer to zero as opposed to specifications with only a few samples and / or wider confidence intervals.

The Which factors GDY and Feminine systematically influenced the magnitude of the summary effect. Specifications that included samples containing gender dysphoric patients or solely "feminine" [13] samples only, produced some of the largest effects. When including GDY as a predictor, the fixed-effect summary estimates for the 52 non-GDY and the 12 GDY samples were $\ln O R=0.05,95 \% \mathrm{CI}=[0.02,0.08]$ and $\ln O R=0.30,95 \% \mathrm{CI}=[0.16,0.44]$. Assuming a random-effects model, these estimates were $\ln O R=$ $0.09,95 \% \mathrm{CI}=[0.03,0.15]$ and $\ln O R=0.31,95 \% \mathrm{CI}=[0.16,0.46]$.

Figure 4 also indicates that as the meta-analytic summary effects increased, so did the frequency of specifications encompassing only those samples, which had already been included in at least one of the previous six meta-analyses [5,7,8,13-16]. Including the Which factor in Previous $M A$ as a moderator in the meta-analysis consisting of all 64 samples, the estimated means were $\ln O R=0.02,95 \% \mathrm{CI}=[-0.02,0.07]$ and $\ln O R=0.11,95 \% \mathrm{CI}=[0.06,0.16]$ (difference of $0.09,95 \% \mathrm{CI}=[0.03,0.15]$ in favour of studies included in previous meta-analyses). The respective random-effects summary estimates were $\ln O R=0.00,95 \% \mathrm{CI}=[-$ $0.13,0.13]$ and $\ln O R=0.15,95 \% \mathrm{CI}=[0.09,0.21]$ (difference of $0.15,95 \% \mathrm{CI}=[-0.01,0.29]$ ).

The meta-analytic specifications across the female samples returned similar results. The summary effects ranged from -0.22 to 0.54 , with an interquartile range of 0.05 to 0.16 . A total of $84.9 \%$ of estimated means 
were greater than 0 of which $32.2 \%$ did not include zero. Again, tighter confidence intervals were observed for summary effects close to zero. No systematic pattern of Which/How factor level combinations was apparent.

Voracek et al. [21] recommended assessing the overall evidence from a specification-curve and multiverse meta-analysis against the respective null model with parametric bootstrapping (inferential specificationcurve plots) and an additional histogram of $p$ values. Here, we extend the idea of the histogram of $p$ values to the $S$-value. A derivation of the distribution of $S$-values under the null model (exponential with rate parameter $\lambda=\ln (2)$ ), with which the observed distribution can be compared, is provided in supplement S7. The inferential specification-curve plots are presented in the supplementary material (S8).

Instead of histograms, Figure 6 (top) displays kernel density estimates of the 1,628 observed $S$ values (orange) and 1,628 random values drawn from an exponential distribution with rate parameter $\lambda=\ln (2)$ (green) as well as the probability density function of said distribution (blue). Clearly, the distribution of the observed $S$-values deviates from both the expected and simulated distribution. Thus, conditional on all statistical background assumptions being met6 [87], a true mean $\ln O R$ of 0 (i.e., the null hypothesis) appears incompatible with the results of the specification curve meta-analysis. We can get an idea of the degree of this incompatibility (or distance) between the data and test model by thinking of the distribution of these $S$-value in terms of surprise. The interquartile range of observed $S$-values was [2.18,6.22], the median $S$-value was 4.02 . That is, under the null model, the central $50 \%$ of results would be less surprising than observing all heads out of seven tosses of a fair coin.

The interquartile range of the distribution of $S$-values for the female samples was 0.73 to 4.47 , with a median of 1.67 .

A specification curve analysis for the difference between the summary estimates in men and women can be found in the supplementary materials.

\subsection{Discussion}

In Part III, we obtained several estimates of the excess of older brothers within the set of older siblings, as reported by homosexual vs. heterosexual men under various conditions and compared these estimates to the same effect in women as a point of reference.

Suppose that the eight meta-analyses of the specific male sample sets summarised in Table 8 had each been put forth by a different researcher. Researcher 1 might argue that only the fixed-effect estimate of the six probability samples should be considered as an adequate summary of the available evidence of the excess of older brothers in homosexual men and conclude that there is not enough information to confidently declare either positive or negative estimates to be incompatible with the available data. Researcher 2 might argue that not the fixed-effect but rather the random-effects summary estimate of the probability samples should be considered, but otherwise agrees with the first researcher's conclusion. Researcher 3 might argue that only the random-effects summary estimate of the 31 samples in [5] should be regarded as a reasonable estimate. Judging by the summary effect's confidence interval, true effects of $9 \%$ and $27 \%$ greater odds for observing an older brother among the older siblings of homosexual men; hence, the effect is most likely positive and thus consistent with the FBOE. While the remaining five researchers disagree with the third researcher's exclusion criteria or model considerations, they largely agree that there is a meaningful positive effect, perhaps not lower than 4\%. All but Researchers 1 and 2 would infer that their results could have only occurred if older brothers indeed increased the odds of homosexual orientation via the MIH.

Researcher 9 might join this imagined conversation, noting that instead of focusing on the estimated summary effects, one should also consider the prediction intervals. Accordingly, Researcher 9 concludes that, taken at face value, all these meta-analyses indicate that both the magnitude and direction of the effect are erratic and that the estimated summary effects are most likely inflated due to small-study effects. Furthermore, the very same methods, which had the six favourable researchers believe in a meaningful excess of older brothers among homosexual men also suggest a similarly sized excess of older brothers among homosexual women.

6 Note that we have reason to believe that these assumptions were not met, as we found evidence for a small sample bias in the available data. 
The results of Part III do not lend to the confident conclusion that older brothers increase the odds of homosexual orientation and thus do not corroborate causal biological theories explaining this increase. The magnitude of the effect is highly uncertain (i.e., either closer to $4 \%$, or closer to $27 \%$ ?) and inconsistent across the available data. Even more important, the comparison of available male and female samples further suggests that homosexual orientation does not account for a large enough chunk of the variation in older brothers beyond variation which may be attributable to an interplay of a host of unknown factors, which in turn might be unrelated to older brothers increasing the odds of homosexual orientation via the process of immunization [92,93]. If the observed effect among women is considered as an estimate of this interplay of unknown factors, it then appears that there is almost no attributable variation left to such explanations.

\subsubsection{How plausible is the fraternal birth-order effect in light of extra-data knowledge?}

If we were to agree with the majority of the nine researchers above and ignore (1) the inconsistency of effects, (2) the apparent disproportionate influence of small samples and (3) the observation of a similarly sized effect in women, we would still be left with the challenge of making sense of the wide range of possible true effects compatible with the available data. We noted earlier that the $O R$ in our analyses can be regarded as the ratio of sex ratios of the subset of older siblings of homosexual and heterosexual men. Few demographic descriptors have been studied as extensively as the human sex ratio [94,95]. As mentioned above, this number frequently is estimated at approximately 1.06 male births per female birth, which is equivalent to a .515 probability of being born male (e.g., [29]). Assuming that this probability is the same for each sibling in a sibship,7 then the sex ratio among the subset of all reported older siblings should also be .515 , regardless of the sexual orientation of the sampled younger brother. 8

The human sex ratio has been found to vary as a function of multiple factors, including, but not limited to, wartime, month of conception, hormonally induced ovulation, artificial insemination, maternal weight, and economic stress [94,96-98]. As for a few examples of these factors, Catalano [98] reported that the collapse of the East German economy in 1991 was associated with a drop of $0.4 \%$ from the expected proportion of male births and that this drop may have been attributable to experienced socio-economic stress in the affected regions. As one of the most extreme deviations, James [94] reported a proportion of .463 males in a relatively small sample of 2,608 births following hormonally induced ovulation. This corresponds to a drop of roughly 5\% from the expected value. Hesketh et al. [99] found that, the sex ratio at birth in China increased from 1.08 in 1981 to 1.16 in 2001, corresponding to a 2.2\% difference in male births during that period. Most notably, these researchers attributed much of this shift to practices of sexselective abortion in this country. It is obvious that such factors have a different causal status in their relationship to the human sex ratio than does homosexual orientation of younger brothers, as the latter certainly cannot cause the sex ratio of older siblings to deviate from the population average. Nevertheless, one may use the extent of such observed variations in the sex ratio, as documented in various research sources [100,101], and then determine where the variation in the sex ratio from the current results would rank among these and make an informed judgment about how plausible such results are.

Assuming a probability of .515 for male birth among the older siblings of heterosexual men, a difference of $0.4 \%$ as in [98] would imply a probability of .519 for male birth among the older siblings of a sample of homosexual younger brothers. We can transform these numbers onto the odds ratio scale, as $O R=$ $0.519 /(1-0.519) \times(1-0.515) / 0.515 \approx 1.02$. Likewise, a difference of 5 percentage points as was observed in the births after hormonally induced ovulation [94] would be equivalent to an OR of 1.22. On the other end, an odds ratio of 1.27 - the highest upper bound of the $95 \%$ confidence intervals of the six favourable researchers' results - would correspond to a difference of $6 \%$. The lowest bound of the confidence intervals

7 This perhaps is too naive an assumption [94], but taking into account within-sibship variation of sex ratios would significantly complicate the interpretation of the current results.

$8 \mathrm{In}$ fact, the random-effects estimate for the average proportion of older brothers among older sisters across all 64 samples of heterosexual men was $0.505,95 \% \mathrm{CI}=[.505, .516]$, as elaborated on in greater detail in the supplementary materials. 
was $O R=1.04$, which would be equivalent to a difference of 1 percentage point. The point-estimate of $O R$ $=1.13$ for the random-effects means across all 64 male samples and the GDY subgroup would correspond to a difference of approximately $3 \%$ and $7 \%$, respectively.

Given these reference values, the (rhetorical) question arises, as to whether one should (or would) believe that the association between the sex ratio of older siblings and the homosexual orientation of a younger brother is comparable in strength to the association between the human sex ratio with either induced ovulation, country-wide sex-selective abortion practices or national economic collapse. What is more, findings from the human sex-ratio literature indicate that the human sex ratio appears to be quite stable under many different circumstances [94,95], with deviations from the expected sex ratio amounting to merely $2 \%$ or more being exceptional and most deviations amounting to merely $1 \%$ or less [100]. Against this empirically based background it appears much more plausible to suppose the true summary effect of the sex ratio among older siblings on their younger brothers' sexual orientation is closer to $O R=1.04$ (i.e., difference of $1 \%$ ) or $O R=1.02$ (i.e., a difference of $0.4 \%$ ) than to $O R=1.27$ (i.e., difference of $6 \%$ ) or even to the random-effects mean of $O R=1.13$ (i.e., difference of $3 \%$ ).

\subsubsection{Why should gender-dysphoric males show an even greater excess of older brothers?}

The specification-curve meta-analyses largely corroborate the findings assembled in Table 8 in that the effect fluctuated across different contexts - for men, as well as in women. The specification-curves also suggested sizeable differences in the summary effect, e.g., between the 11 samples comprised of male participants diagnosed with GDY versus the 55 remaining samples. Subgroup analyses - when taken at face value - would have researchers believe that the odds of observing an older brother among the older siblings of genderdysphoric homosexual men are compatible with averages of between $16 \%$ and $61 \%$ greater odds of observing an older brother among older siblings than those same odds for the older sibings reported by the control group. In comparison, the summary effect for the older siblings of non-GDY homosexual men would be regarded as compatible with a range of $2 \%$ and $16 \%$. Three of the GDY samples employed behavioural measures to classify sexual orientation. In these samples, participants were categorized according to Blanchard's retrospective interpretation of their medical charts [48] or prospectively through the assessment of "feminine" behaviours of children and adolescents [51,76]. Two GDY samples [2,56] did not assess sexual orientation at all, but used GDY diagnoses as a proxy for homosexual orientation. In addition, five of the GDY samples [2,48,60,67] employed Blanchard's [102] typology for classifying gender dysphoric individuals as homo- versus nonhomosexual/autogynephilic. The validity of this typology is disputed (e.g., [103]). This inconsistency with respect to the classification of sexual orientation may help to account for the relatively large but uncertain summary effect in the GDY samples. For instance, Blanchard et al. [15] and VanderLaan et al. [76] remarked that the prospective classification of children as homosexual and heterosexual will inadvertently result in the missclassification of some individuals. However, their conclusion that any differences between groups that manage to be statistically significant despite the extra noise brought about by the greater error in the assessment of sexual orientation should be regarded as strong evidence that such a difference is incorrect [104]. Given the many researcher degrees of freedom, it is generally not difficult to obtain a statistically significant difference between any groups [46]. However, for the effect estimate to become statistically significant at the 5\% level it must fall at least two standard errors away from zero. Greater measurement error implies greater standard errors and thus an estimate must be relatively far from zero to reach the significance threshold [100,104]. The true difference between groups may however be much closer to zero than the statistically significant difference would indicate. As a consequence, observed differences between groups is likely overestimate any underlying true difference [101]. Similarly, one study [72] compared the number of older brothers of participants who had been formally diagnosed with GDY to a non-GDY control group - importantly, the sexual orientation of these participants was never assessed. Thus if researchers are willing to treat [72] as evidence for the FBOE they are essentially treating GDY and non-GDY as proxies for homosexual and heterosexual orientation, respectively. This deviates from numerous other study designs (e.g., $[48,51]$ ) by the same group of authors (Blanchard et al.), wherein the sexual orientation of GDY participants was assessed and the difference in the number of older brothers between homosexual versus nonhomosexual GDY-participants was interpreted as evidence for the FBOE. Thus, the same group of researchers appears to uphold contradictory convictions about how the FBOE should be investigated in GDY samples: On the one hand, a GDY diagnosis is regarded as a valid proxy for homosexual orientation, 
on the other hand the meticulous distinction between nonhomosexual and homosexual GDYs must be made in order to detect a difference in the number of older brothers.

Overall, there seems to be a tremendous degree of heterogeneity regarding the validity of classification schemes, accompanied by noisy measurements and the overestimation of the excess of older brothers in one group versus the other. It is unsurprising that such samples would be associated with larger and more erratic effects as opposed to samples with less measurement error and more similar classification schemes.

\section{General discussion and conclusion}

This work set out to re-examine the statistical and empirical foundation of the FBOE by triangulating basic eivdence from probability calculus (part I), data simulation (part II) and specification-curve and multiverse meta-analysis (part III). In parts I and II we showed that currently recommended and widely used measures for quantifying the putative excess of older brothers in homosexual men rest on the false assumptions that statistical models used to investigate this effect (a) need to control for the confounding effect of total family size and (b) that this can be achieved through the use of ratio variables. Furthermore, we re-emphasised the importance of controlling for the number of older siblings, when investigating the putative excess of older brothers, a necessary observation for the FBOE. In part III we re-assessed the metaanalytic evidence for an excess of older brothers among homosexual men using an adequate effect size metric and carried out the crucial comparison between male and female samples.

Evidently, the sibship data from over 30,000 homosexual men and women were not enough to estimate the size of this difference to a range of values, which would permit confident inferences about its direction or magnitude. Across male samples, we also found evidence for a disproportionate influence of smaller samples on meta-analytic summary estimates. Such a pattern frequently signals publication bias and suggests that the meta-analytic summary estimates should be adjusted towards zero. A series of specification-curve and multiverse meta-analyses suggested an implausibly large and highly uncertain excess of older brothers in samples comprising individuals diagnosed with GDY, which, upon closer examination, are expected to provide overestimates of any excess of older brothers, due to their inherently noisier classification of sexual orientation. Altogether, the current lines of results suggest that if there is any true excess of older brothers among homosexual men, the available data do not contain enough information to permit a meaningfully precise estimation of its magnitude. This uncertainty, together with the inconsistency (or variability) of the excess of older brothers among homosexual men, as well as women, overshadows meaningful signals in the currently available data sources that might be attributable to the FBOE or the MIH.

We reiterate, that the current results - but also those of [5], [13] and [8] - are predicated on the assumption that reported siblings can be regarded as indpendent observations. However, given the retrievable data, our analyses represent the most thorough and methodologically sound investigation of the FBOE and the MIH to date. The reason we had to work with reported siblings as the observations rather than the participants or individuals whose data were actually recorded, lies in the systematic lack of reporting of basic summary statistics in the corpus of primary studies on the FBOE. Almost half of the means and standard deviations in Table 5 were not retrievable from the information provided in the respective primary publications, rendering their analyses and results irreprocucible. Moreover, not a single data set on the FBOE has been made publicly available yet (the data set presented in the current study is the first one).

In light of all of the above, an excess of older brothers in homosexual men seems to have been born out of the continued misapplication of erroneous statistical methods (controlling for the wrong confounder and using ratio variables). We recommend that researchers interested in the FBOE should instead adopt contemporary practices of open science [105] and use adequate analysis plans, which control for the number of older siblings. This will contribute towards improving the quality and validity of FBOE research.

\section{Author's Contributions}

UST conceived the study idea and provided original data. JKV developed the formalisms in Part I, conducted the simulation study in Part II, collected the meta-analytic data and carried out the metaanalyses in Part III, and wrote the first draft of the manuscript. MK provided critical revision of the 
analysis code, the statistical analyses, and the manuscript. MV and UST supervised the work and provided critical revision of the statistical analyses and the manuscript. MV and UST suggested conducting publication bias analyses and MV the use of the Albatross plot. All authors approved the final version of the manuscript.

\section{Acknowledgements}

We thank Anton Rupprecht for his contributions in preparatory stages of this study. 


\section{References}

1. Blanchard R, Bogaert AF. 1996

Biodemographic comparisons of homosexual and heterosexual men in the Kinsey interview data. Arch. Sex. Behav. 25 551-579. (doi:10.1007/BF02437839) 2. Blanchard R, Bogaert AF. 1996 Homosexuality in men and number of older brothers. Am. J. Psychiat. 153, 27-31. (doi:10.1176/ajp.153.1.27)

3. James WH. 2004 The cause(s) of the fraternal birth order effect in male homosexuality. J. Biosoc. Sci. 36, 51-59. (doi:10.1017/S0021932004006066) 4. Balthazart J. 2018 Fraternal birth order effect on sexual orientation explained. Proc. Natl. Acad. Sci. U.S.A. 115, 234-236. (doi:10.1073/pnas.1719534115) 5. Blanchard R. 2018 Fraternal birth order, family size, and male homosexuality: Meta-Analysis of studies spanning 25 years. Arch. Sex. Behav. 47, $1-$ 15. (doi:10.1007/s10508-017-1007-4) $6 . \quad$ Bogaert AF. 2006 Biological versus nonbiological older brothers and men's sexual orientation. Proc. Natl. Acad. Sci. USA 103, 10771-10774. (doi:10.1073/pnas.0511152103) 7. Blanchard R. 2004 Quantitative and theoretical analyses of the relation between older brothers and homosexuality in men. J. Theor. Biol. 230, 173-187. (doi:10.1016/j.jtbi.2004.04.021) 8. Blanchard R, Beier KM, Gómez Jiménez FR, Grundmann D, Krupp J, Semenyna SW, Vasey PL. 2021 MetaAnalyses of Fraternal and Sororal Birth Order Effects in Homosexual Pedophiles, Hebephiles, and Teleiophiles. Arch. Sex. Behav. 50, 779-796. (doi:10.1007/s10508020-01819-3)

\section{Blanchard R. 2001 Fraternal}

birth order and the maternal immune hypothesis of male homosexuality. Horm. Behav. 40, 105-114.

(doi:10.1006/hbeh.2001.1681)

10. Bogaert AF, Skorska M. 2011 Sexual orientation, fraternal birth order, and the maternal immune hypothesis: $\mathrm{A}$ review. Front. Neuroendocrinol. 32, 247254. (doi:10.1016/j.yfrne.2011.02.004) $11 . \quad$ Bogaert AF, Skorska MN, Wang C, Gabrie J, MacNeil AJ, Hoffarth MR, VanderLaan DP, Zucker KJ, Blanchard R. 2018 Male homosexuality and maternal immune responsivity to the Y-Linked protein NLGN4Y. Proc. Natl. Acad. Sci. USA 115, 302-306.

(doi:10.1073/pnas.1705895114)

12. Blanchard R, Lippa RA. 2021 Reassessing the Effect of Older Sisters on Sexual Orientation in Men. Arch. Sex. Behav. 50, 797-805. (doi:10.1007/s10508020-01840-6)

13. Blanchard R. 2018 Response to commentaries: Meta-Analysis of probability samples and other new evidence. Arch. Sex. Behav. 47, 49-57. (doi:10.1007/s10508017-1134-y)

$14 . \quad J o n e s$ MB, Blanchard R. 1998 Birth order and male homosexuality:
Extension of Slater's index. Hum. Biol. 70, 775-787.

15. Blanchard R, VanderLaan DP. 2015 Commentary on Kishida and Rahman (2015), including a meta-analysis of relevant studies on fraternal birth order and sexual orientation in men. Arch. Sex. Behav. 44, 1503-1509.

(doi:10.1007/s10508-015-0555-8)

16. Blanchard R, Krupp J,

VanderLaan DP, Vasey PL, Zucker KJ. 2020 A method yielding comparable estimates of the fraternal birth order and female fecundity effects in male homosexuality.

Proc. R. Soc. B-Biol. Sci. 287, 20192907. (doi:10.1098/rspb.2019.2907)

17. Blanchard R. 2018 Older brothers and older sisters odds ratios in 36 samples of homosexual males. Arch. Sex. Behav. 47, 829-832. (doi: $10.1007 /$ s10508018-1160-4)

$18 . \quad$ Blanchard R. 2020 Comparison of two methods for studying fraternal birth order and homosexuality in men. Arch. Sex. Behav. 49, 57-60. (doi:10.1007/s10508019-01553-5)

19. Blanchard R. 2014 Detecting and correcting for family size differences in the study of sexual orientation and fraternal birth order. Arch. Sex. Behav. 43 845-852. (doi:10.1007/s10508-013-0245-3) 20. Gigerenzer G. 2018 Statistical rituals: The replication delusion and how we got there. Adv. Methods Pract. Psychol. Sci. 1, 198-218.

(doi: 10.1177/2515245918771329)

21. Voracek M, Kossmeier M, Tran US. 2019 Which data to meta-analyze, and how? A specification-curve and multiverseanalysis approach to meta-analysis. $Z$. Psychol. 227, 64-82. (doi: $10.1027 / 2151-$ 2604/a000357)

$22 . \quad$ Simonsohn U, Simmons JP, Nelson LD. 2020 Specification curve analysis. Nat. Hum. Behav. 4, 1208-1214. (doi:10.1038/s41562-020-0912-z) 23. Steegen S, Tuerlinckx F, Gelman A, Vanpaemel W. 2016 Increasing transparency through a multiverse analysis. Perspect. Psychol. Sci. 11, 702-712. (doi:10.1177/1745691616658637) 24. Apostolou M. 2020 Does fraternal birth order predict male homosexuality, bisexuality, and heterosexual orientation with same-sex attraction? Evidence from a GreekSpeaking sample from Greece. Arch. Sex. Behav. 49, 575-579. (doi:10.1007/s10508019-01466-3)

25. Skorska MN, Bogaert AF. 2020

Fraternal birth order, only-child status, and sibling sex ratio related to sexual orientation in the add health data: A reanalysis and extended findings. Arch. Sex. Behav. 49, 557-573. (doi:10.1007/s10508019-01496-x)

26. Blitzstein JK, Hwang J. 2019

Introduction to probability (2nd ed). Boca Raton: FL. Taylor \& Francis.

27. Simpson EH. 1951 The

interpretation of interaction in contingency tables. J. R. Stat. Soc. Series B. Stat. Methodol. 13, 238-241.

28. Good PI, Hardin JW. 2012 Common errors in statistics (and how to avoid them) (4th ed.). Hoboken, NJ: Wiley. 29. Grech V, Mamo J. 2020 What is the sex ratio at birth? Early Hum. Dev. 140, 104858.

(doi:10.1016/j.earlhumdev.2019.104858)

30. Zietsch BP. 2018 Reasons for caution about the fraternal birth order effect. Arch. Sex. Behav. 47, 47-48. (doi:10.1007/s10508-017-1086-2) 31. Gelman A, Stern H. 2006 The difference between 'Significant' and 'Not significant' is not itself statistically significant. Am. Stat. 60, 328-331. (doi:10.1198/000313006X152649) 32. Sollberger S, Ehlert U. 2016 How to use and interpret hormone ratios. Psychoneuroendocrinology 63, 385-397. (doi:10.1016/j.psyneuen.2015.09.031) 33. Kronmal RA. 1993 Spurious correlation and the fallacy of the ratio standard revisited. J. R. Stat. Soc. Ser. A Stat. Soc. 156, 379. (doi:10.2307/2983064) 34. Wiseman RM. 2009 On the use and misuse of ratios in strategic management research. In Research Methodology in Strategy and Management, pp. 75-110. (doi:10.1108/S14798387(2009)0000005004)

35. Frisch M, Hviid A. 2006 Childhood family correlates of heterosexual and homosexual marriages: A national cohort study of two million Danes. Arch. Sex. Behav. 35, 533-547.

(doi:10.1007/s10508-006-9062-2) 36. $R$ Core Team. $2020 \mathrm{R}: \mathrm{A}$ language and environment for statistical computing. https://www.R-project.org/. 37. Greenland S, Chow ZR. 2019 To Aid Statistical Inference, Emphasize Unconditional Descriptions of Statistics. https://arxiv.org/abs/1909.08583. 38. Goldfeld K, Wujciak-Jens J. 2020 Simstudy: Simulation of study data. See https://CRAN.R-

project.org/package=simstudy. 39. Gaujoux R. 2020 doRNG: Generic reproducible parallel backend for 'foreach' loops. See https://CRAN.Rproject.org/package $=$ doRNG. 40. Microsoft, Weston S. 2019 doParallel: Foreach parallel adaptor for the 'parallel' package. See https://CRAN.Rproject.org/package=doPrallel.

41. Microsoft, Weston S. 2020 Foreach: Provides foreach looping construct. See https://CRAN.Rproject.org/package=foreach. 42. Cantor JM, Blanchard R, Paterson AD, Bogaert AF. 2002 How many gay men owe their sexual orientation to fraternal birth order? Arch. Sex. Behav. 31, 63-71. (doi:10.1023/A:1014031201935) $43 . \quad$ Blanchard R, Zucker KJ, CohenKettenis PT, Gooren LG, Bailey JM. 1996 Birth order and sibling sex ratio in two samples of Dutch gender-dysphoric 
homosexual males. Arch. Sex. Behav. 25, 495-514. (doi:10.1007/BF02437544)

44. Blanchard R, Klassen P. $1997 \mathrm{H}$

$\mathrm{Y}$ antigen and homosexuality in men. $J$.

Theor. Biol. 185, 373-378.

(doi:10.1006/jtbi.1996.0315)

45. Tran US, Kossmeier M, Voracek

M. 2019 Associations of bisexuality and

homosexuality with handedness and

footedness: A latent variable analysis

approach. Arch. Sex. Behav. 48, 1451-1461.

(doi: $10.1007 /$ s10508-018-1346-9)

46. Simmons JP, Nelson LD

Simonsohn U. 2011 False-positive

psychology: Undisclosed flexibility in data

collection and analysis allows presenting

anything as significant. Psychol. Sci. 22,

1359-1366.

(doi:10.1177/0956797611417632)

47. Gelman A, Loken E. 2013 The

garden of forking paths: Why multiple

comparisons can be a problem, even when

there is no 'Fishing Expedition' or ' $p$ -

Hacking' and the research hypothesis was

posited ahead of time. Unpublished

Manuscript. See

http://www.stat.columbia.edu/ gelman/re search/unpublished/p hacking.pdf.

$48 . \quad$ Blanchard R, Sheridan P M.

1992 Sibship size, sibling sex ratio, birth

order, and parental age in homosexual and

nonhomosexual gender dysphorics. J. Nerv.

Ment. Dis. 180, 40-47.

49. Blanchard R, Zucker KJ. 1994

Reanalysis of Bell, Weinberg, and

Hammersmith's data on birth order,

siblings sex ratio, and parental age in

homosexual men. Am. J. Psychiatry 151,

1375-1376.

50. Zucker KJ, Blanchard R. 1994

Reanalysis of Bieber et al.'s 1962 data on

sibling sex ratio and birth order in male

homosexuals. J. Nerv. Ment. Dis. 182, 528-

530.

51. Blanchard R, Zucker KJ, Bradley

SJ, Hume CS. 1995 Birth order and sibling

sex ratio in homosexual male adolescents

and probably prehomosexual feminine

boys. Dev. Psychol. 31, 22-30.

52. Bogaert AF, Bezeau S, Kuban M

Blanchard R. 1997 Pedophilia, sexual

orientation, and birth order. J. Abnorm.

Psychol. 106, 331-335. (doi:10.1037/0021843X.106.2.331)

53. Blanchard R, Bogaert AF. 1998

Birth order in homosexual versus

heterosexual sex offenders against

children, pubescents, and adults. Arch. Sex.

Behav. 27, 595-603.

54. Ellis L, Blanchard R. 2001 Birth

order, sibling sex ratio, and materna

miscarriages in homosexual and

heterosexual men and women. Personal.

Individ. Differ. 30, 543-552.

(doi:10.1016/S0191-8869(00)00051-9)

55. Blanchard R, Cantor JM

Bogaert AF, Breedlove SM, Ellis L. 2006

Interaction of fraternal birth order and

handedness in the development of male

homosexuality. Horm. Behav. 49, 405-414.

(doi:10.1016/j.yhbeh.2005.09.002)

56. Blanchard R, Kuban ME, Blak T, Klassen PE, Dickey R, Cantor JM. 2012

Sexual Attraction to Others: A Comparison

of Two Models of Alloerotic Responding in

Men. Arch Sex Behav 41, 13-29.

(doi:10.1007/s10508-010-9675-3)

57. Bogaert AF. 2010 Physical

development and sexual orientation in men and women: An analysis of NATSAL-2000. Arch. Sex. Behav. 39, 110-116.

(doi:10.1007/s10508-008-9398-x)

58. Zietsch BP, Verweij KJH, Heath

AC, Madden PAF, Martin NG, Nelson EC,

Lynskey MT. 2012 Do shared etiological

factors contribute to the relationship

between sexual orientation and

depression? Psychol. Med. 42, 521-532.

(doi:10.1017/S0033291711001577)

59. Camperio-Ciani A, lemmola $F$,

Blecher SR. 2009 Genetic factors increase

fecundity in female maternal relatives of

bisexual men as in homosexuals. J. Sex.

Med. 6, 449-455. (doi:10.1111/j.1743-

6109.2008.00944.x)

60. Green R. 2000 Birth order and

ratio of brothers to sisters in transsexuals.

Psychol. Med. 30, 789-795.

(doi:10.1017/S0033291799001932)

61. Yule MA, Brotto LA, Gorzalka

BB. 2014 Biological markers of asexuality:

Handedness, birth order, and finger length

ratios in self-identified asexual men and

women. Arch. Sex. Behav. 43, 299-310.

(doi:10.1007/s10508-013-0175-0)

62. Blanchard R, Lippa RA. 2007

Birth order, sibling sex ratio, handedness,

and sexual orientation of male and female

participants in a BBC internet research

project. Arch. Sex. Behav. 36, 163-176.

(doi:10.1007/s10508-006-9159-7)

63. Bogaert AF. 2005 Sibling sex

ratio and sexual orientation in men and

women: New tests in two national

probability samples. Arch. Sex. Behav. 34,

111-116. (doi:10.1007/s10508-005-1005-9)

64. Xu Y, Zheng Y. 2014 Birth order

and sibling sex ratio in relation to sexual

orientation in China. Soc. Behav. Personal.

Int. J. 42, 995-1001.

(doi: $10.2224 / \mathrm{sbp} .2014 .42 .6 .995)$

65. Kangassalo K, Pölkki M, Rantala

MJ. 2011 Prenatal influences on sexual

orientation: Digit ratio (2D:4D) and number

of older siblings. Evol. Psychol. 9

147470491100900.

(doi: $\frac{10.1177 / 147470491100900402)}{X u Y, Z h e n g Y .2017 \text { Fratern }}$

66. Xu Y, Zheng Y. 2017 Fraternal

birth order, handedness, and sexual

orientation in a Chinese population. J. Sex

Res. 54, 10-18.

(doi:10.1080/00224499.2015.1104530)

67. Gómez-Gil E, Esteva I, Carrasco

$R$, Almaraz MC, Pasaro E, Salamero M,

Guillamon A. 2011 Birth order and ratio of

brothers to sisters in Spanish transsexuals.

Arch. Sex. Behav. 40, 505-510.

(doi:10.1007/s10508-010-9614-3)

68. Semenyna SW, VanderLaan DP,

Vasey PL. 2017 Birth order and recalled

childhood gender nonconformity in

Samoan men and Fa'afafine. Dev.

Psychobiol. 59, 338-347.

(doi: $10.1002 /$ dev.21498)

69. Vanderlaan DP et al. 2017 Birth

order and androphilic male-to-female

transsexualism in Brazil. J. Biosoc. Sci. 49,

527-535.

(doi:10.1017/S0021932016000584)

70. VanderLaan DP, Vasey PL. 2011

Male sexual orientation in independent

Samoa: Evidence for fraternal birth order

and maternal fecundity effects. Arch. Sex.

Behav. 40, 495-503. (doi:10.1007/s10508009-9576-5)

71. Vasey PL, VanderLaan DP. 2007

Birth order and male androphilia in Samoan
Fa'afafine. Proc. R. Soc. B Biol. Sci. 274,

1437-1442. (doi:10.1098/rspb.2007.0120)

72. Schagen SEE, Delemarre-van de

Waal HA, Blanchard R, Cohen-Kettenis PT.

2012 Sibling sex ratio and birth order in

early-onset gender dysphoric adolescents.

Arch. Sex. Behav. 41, 541-549.

(doi:10.1007/s10508-011-9777-6)

73. Viechtbauer W. 2010

Conducting meta-analyses in $\mathrm{R}$ with the

metafor package. J. Stat. Softw. 36, 1-48. (doi:10.18637/jss.v036.i03)

74. Van den Noortgate W, López-

López JA, Marín-Martínez F, Sánchez-Meca

J. 2013 Three-level meta-analysis of

dependent effect sizes. Behav Res 45, 576-

594. (doi:10.3758/s13428-012-0261-6)

75. Blanchard R, Barbaree $\mathrm{HE}$

Bogaert AF, Dickey R, Klassen P, Kuban ME,

Zucker KJ. 2000 Fraternal birth order and

sexual orientation in pedophiles. Arch. Sex.

Behav. 29, 463-478.

76. VanderLaan DP, Blanchard R,

Wood H, Zucker KJ. 2014 Birth order and

sibling sex ratio of children and adolescents

referred to a gender identity service. PLOS

ONE 9, e90257.

(doi:10.1371/journal.pone.0090257)

77. Hedges LV, Vevea JL. 1998

Fixed- and random-effects models in meta-

analysis. Psychol. Methods 3, 486-504.

(doi:10.1037/1082-989X.3.4.486)

$78 . \quad$ Rice K, Higgins JPT, Lumley T.

2018 A re-evaluation of fixed effect(s)

meta-analysis. J. R. Stat. Soc. A 181, 205-

227. (doi:10.1111/rssa.12275)

$79 . \quad$ Higgins JPT, Thompson SG,

Spiegelhalter DJ. 2009 A re-evaluation of

random-effects meta-analysis. J. R. Stat.

Soc. Ser. A Stat. Soc. 172, 137-159.

(doi:10.1111/j.1467-985X.2008.00552.x)

80. Silberzahn R et al. 2018 Many

Analysts, One Data Set: Making

Transparent How Variations in Analytic

Choices Affect Results. Adv. Meth. Pract.

Psychol. Sci. 1, 337-356.

(doi: $\frac{10.1177 / 2515245917747646)}{X u Y, \text { ) }}$

81. Xu Y, Norton S, Rahman Q.

2019 Early life conditions and adolescent

sexual orientation: A prospective birth

cohort study. Dev. Psychol. 55, 1226-1243.

(doi:10.1037/dev0000704)

82. Kossmeier M, Tran US, Voracek

M. 2020 Metaviz: Forest plots, funnel plots,

and visual funnel plot inference for meta-

analysis. See https://CRAN.R-

project.org/package=metaviz.

83. Wickham H et al. 2019

Welcome to the Tidyverse. J. Open Source

Softw. 4, 1686. (doi:10.21105/joss.01686)

84. Morey RD, Hoekstra R, Roude

JN, Lee MD, Wagenmakers E-J. 2016 The

fallacy of placing confidence in confidence

intervals. Psychon. Bull. Rev. 23, 103-123.

(doi:10.3758/s13423-015-0947-8)

85. Hardy RJ, Thompson SG. 1996 A

likelihood approach to meta-analysis with

random effects. Stat. Med. 15, 619-629.

(doi:10.1002/(SICI)1097-

0258(19960330)15:6<619::AID-

SIM188>3.0.CO;2-A)

$86 . \quad$ Rafi Z, Greenland S. 2020

Semantic and cognitive tools to aid

statistical science: Replace confidence and

significance by compatibility and surprise.

BMC Med.I Res. Methodol. 20, 244. (doi:

doi.org/10.1186/s12874-020-01105-9) 
values behave exactly as they should: Some misleading criticisms of $p$-values and their resolution with S-values. Am. Stat. 73, 106114

(doi:10.1080/00031305.2018.1529625)

88. Schild AHE, Voracek M. 2015

Finding your way out of the forest without a trail of bread crumbs: Development and evaluation of two novel displays of forest plots: Evaluation of novel designs of forest plots. Res. Synth. Methods 6, 74-86.

(doi:10.1002/jrsm.1125)

89. Vevea JL, Coburn K, Sutton A. 2019 Publication bias. In the handbook of research synthesis and meta-analysis (eds $\mathrm{H}$ Cooper, LV Hedges, JC Valentine), pp. 383432. New York: Russell Sage Foundation. 90. Egger M, Davey Smith G, Schneider M, Minder C. 1997 Bias in metaanalysis detected by a simple, graphical test. BMJ 315, 629-634.

91. Harrison S, Jones HE, Martin RM, Lewis SJ, Higgins JPT. 2017 The albatross plot: A novel graphical tool for presenting results of diversely reported studies in a systematic review. Res. Synth. Methods 8, 281-289.

(doi:10.1002/jrsm.1239)

92. Meehl PE. 1990 Why

summaries of research on psychological

theories are often uninterpretable. Psychol. Rep. 66, 195-244.

93. Yarkoni T. 2019 The

generalizability crisis. Behav. Brain Sci. 1-37 (doi: (doi:10.1017/S0140525X20001685)

94. James WH. 1987 The human

sex ratio. Part 1: A review of the literature. Hum. Biol. 59, 721-752.

95. Chahnazarian A. 1988

Determinants of the sex ratio at birth:

Review of recent literature. Biodemogr.

Soc. Biol. 35, 214-235.

(doi:10.1080/19485565.1988.9988703)

96. Cagnacci A, Renzi A, Arangino S,

Alessandrini C, Volpe A. 2003 The male

disadvantage and the seasonal rhythm of sex ratio at the time of conception. Hum

Reprod. 18, 885-887.

(doi:10.1093/humrep/deg185)

97. Cagnacci A, Renzi A, Arangino S, Alessandrini C, Volpe A. 2004 Influences of maternal weight on the secondary sex ratio of human offspring. Human Reproduction 19, 442-444.

(doi:10.1093/humrep/deh071)

98. Catalano RA. 2003 Sex ratios in the two Germanies: A test of the economic stress hypothesis. Hum Reprod 18, 19721975. (doi:10.1093/humrep/deg370) 99. Hesketh T, Xing ZW. 2006 Abnormal sex ratios in human populations: Causes and consequences. Proc. Natl. Acad. Sci. USA 103, 13271-13275.

(doi:10.1073/pnas.0602203103)

100. Gelman A, Weakliem D. 2009

Of Beauty, Sex and Power: Too little attention has been paid to the statistical challenges in estimating small effects. Am. Sci. 97, 310-316. (doi:10.2307/27859361) 101. Gelman A, Carlin J. 2014 Beyond power calculations: Assessing type $\mathrm{S}$ (sign) and type $\mathrm{M}$ (magnitude) errors. Perspect. Psychol. Sci. 9, 641-651.

(doi:10.1177/1745691614551642) 102. Blanchard R. 1989 The classification and labeling of nonhomosexual gender dysphorias. Arch. Sex. Behav. 18, 315-334.

(doi:10.1007/BF01541951)

103. Serano JM. 2010 The case against autogynephilia. Int. J.

Transgenderism 12, 176-187. (doi:10.1080/15532739.2010.514223)

104. Loken E, Gelman A. 2017

Measurement error and the replication crisis. Science 355, 584-585.

(doi:10.1126/science.aal3618)

105. Munafò MR et al. 2017 A

manifesto for reproducible science. Nat Hum. Behav. 1, 0021. (doi:10.1038/s41562016-0021)

106.

Blanchard R, Zucker KJ

Siegelman M, Dickey R, Klassen P. 1998 The relation of birth order to sexual orientation in men and women. J. Biosoc. Sci. 30, 511519. (doi:10.1017/S0021932098005112)

107. Rahman Q Wilson GD

Abrahams S. 2004 Biosocial factors, sexual orientation and neurocognitive functioning. Psychoneuroendocrinology 29, 867-881. (doi:10.1016/S0306-4530(03)00154-9)

108. King M, Green J, Osborn DPJ, Arkell J, Hetherton J, Pereira E. 2005 Family size in white gay and heterosexual men.

Arch. Sex. Behav. 34, 117-122.

(doi:10.1007/s10508-005-1006-8)

109. Rahman Q. 2005 Fluctuating asymmetry, second to fourth finger length ratios and human sexual orientation. Psychoneuroendocrinology 30, 382-391. (doi:10.1016/j.psyneuen.2004.10.006)

110. Zucker KJ, Blanchard R, Kim T-S, Pae C-U, Lee C. 2007 Birth order and sibling sex ratio in homosexual transsexual South Korean men: Effects of the male-preference stopping rule. Psychiatry Clin. Neurosci. 61, 529-533. (doi:10.1111/j.1440-

\subsubsection{3.x)}

111. Rahman Q, Collins A, Morrison M, Orrells JC, Cadinouche K, Greenfield S, Begum S. 2008 Maternal inheritance and familial fecundity factors in male homosexuality. Arch. Sex. Behav. 37, 962 969. (doi:10.1007/s10508-007-9191-2)

112. Rahman Q, Clarke K, Morera T. 2009 Hair whorl direction and sexual orientation in human males. Behav. Neurosci. 123, 252-256. (doi:10.1037/a0014816)

113. Schwartz G, Kim RM, Kolundzija $A B$, Rieger G, Sanders AR. 2010 Biodemographic and physical correlates of sexual orientation in men. Arch. Sex. Behav.
39, 93-109. (doi:10.1007/s10508-0099499-1)

114. Blanchard R, Beier KM, Gómez

Jiménez FR, Grundmann D, Krupp J,

Semenyna SW, Vasey PL. 2020 Meta-

analyses of fraternal and sororal birth order effects in homosexual pedophiles,

hebephiles, and teleiophiles. Arch. Sex.

Behav. (doi:10.1007/s10508-020-01819-3)

$115 . \quad$ Bozkurt A, Bozkurt OH, Sonmez

I. 2015 Birth order and sibling sex ratio in a population with high fertility: Are Turkish male to female Transsexuals different? Arch. Sex. Behav. 44, 1331-1337. (doi:10.1007/s10508-014-0425-9)

116. Currin JM, Gibson L, Hubach RD. 2015 Multidimensional assessment of sexual orientation and the fraternal birth order effect. Psychol. Sex. Orientat. Gend. Divers. 2, 113-122.

(doi:10.1037/sgd0000103)

117. Kishida M, Rahman Q. 2015

Fraternal birth order and extreme righthandedness as predictors of sexual orientation and gender nonconformity in men. Arch. Sex. Behav. 44, 1493-1501. (doi:10.1007/s10508-014-0474-0) 118. Austin SW. 2017 Fertility and reproduction's niche: Human sexual diversity. Unpublished master's thesis, University of Montana, Missoula, MT. 119. Swift-Gallant A, Coome LA, Monks DA, VanderLaan DP. 2018 Gender nonconformity and birth order in relation to anal sex role among gay men. Arch. Sex. Behav. 47, 1041-1052.

(doi:10.1007/s10508-017-0980-y)

$120 . \quad$ Nila S, Crochet P-A, Barthes J, Rianti P, Juliandi B, Suryobroto B, Raymond M. 2019 Male homosexual preference:

Femininity and the older brother effect in indonesia. Evol. Psychol. 17, 147470491988070.

(doi:10.1177/1474704919880701)

121. Gómez Jiménez FR, Semenyna

SW, Vasey PL. 2020 The relationship between fraternal birth order and childhood sex-atypical behavior among the Istmo Zapotec muxes. Dev. Psychobiol. 62 792-803. (doi:10.1002/dev.21987)

122. Khorashad BS, Zucker KJ, Talaei A, Rajabzadeh F, Hamed Z, Akbarian P, Blanchard R. 2020 Birth order and sibling sex ratio in androphilic males and gynephilic females diagnosed with gender dysphoria from Iran. J. Sex. Med. 17, 11951202. (doi:10.1016/j.jsxm.2020.02.004) 123. Skorska MN, Coome LA, Saokhieo P, Kaewthip O, Chariyalertsak S, VanderLaan DP. 2020 Handedness and birth order among heterosexual men, gay men, and Sao Praphet Song in Northern Thailand. Arch. Sex. Behav. (doi: 10.1007/s10508-020-01774-z) 
Tables 
Table 1. Overview of previous meta-analyses on the excess of older brothers in homosexual men.

\begin{tabular}{|c|c|c|c|c|c|c|}
\hline Authors & Year & \#Samples & Group & $\mathrm{N}$ & Effect Size & Goal \\
\hline $\begin{array}{l}\text { Jones and } \\
\text { Blanchard } \\
{[14]}\end{array}$ & 1998 & 9 & Homo & 827 & $\begin{array}{l}\text { Fraternal- } \\
\text { and sororal } \\
\text { indices }\end{array}$ & $\begin{array}{l}\text { Primary goal was to determine whether older sisters } \\
\text { showed any association with homosexual orientation } \\
\text { in men. [5] }\end{array}$ \\
\hline & & & Hetero & 2,115 & & \\
\hline \multirow[t]{2}{*}{ Blanchard [7] } & 2004 & 14 & Homo & 3,181 & none & $\begin{array}{l}\text { Each of } 28 \text { homo- and heterosexual groups ( } 14 \\
\text { samples) was treated as an independent observation. } \\
P \text {-value of change in logistic regression's deviance } \\
\text { associated with the removal of the samples' average } \\
\text { number of older brothers from the list of predictors } \\
\text { was statistically significant and therefore interpreted } \\
\text { as supporting the FBOE. }\end{array}$ \\
\hline & & & Hetero & 6,962 & & \\
\hline \multirow[t]{2}{*}{$\begin{array}{l}\text { Blanchard } \\
\text { and } \\
\text { VanderLaan } \\
{[15]}\end{array}$} & 2015 & 14 & Homo & NA & none & $\begin{array}{l}\text { Sign-test meta-analysis over selection of samples not } \\
\text { collected by Blanchard or VanderLaan or any other } \\
\text { frequent collaborators to mitigate the potential of } \\
\text { experimenter (or lab) bias }\end{array}$ \\
\hline & & & Hetero & NA & & \\
\hline \multirow[t]{2}{*}{ Blanchard [5] } & 2018 & 30 & Homo & 7,140 & $O B O R$ & $\begin{array}{l}\text { Three primary stated goals: (1) Assess the effect of } \\
\text { family size on the FBOE, (2) assess whether the } \\
\text { magnitude of the FBOE is stronger in "feminine" as } \\
\text { opposed to more "masculine" samples, (3) update } \\
\text { previous meta-analyses and examine the reliability of } \\
\text { the FBOE. }\end{array}$ \\
\hline & & & Hetero & 12,837 & & \\
\hline \multirow[t]{2}{*}{$\begin{array}{l}\text { Blanchard } \\
{[13]}\end{array}$} & 2018 & 6 & Homo & 3,386 & OBOR & $\begin{array}{l}\text { Primary goal was to respond to Zietsch [30] who } \\
\text { questioned the in- and exclusion criteria in [5], } \\
\text { resulting in the exclusion of all available probability } \\
\text { samples. Blanchard [13] thus conducted a meta- } \\
\text { analysis over six probability samples. }\end{array}$ \\
\hline & & & Hetero & 445,301 & & \\
\hline \multirow[t]{2}{*}{$\begin{array}{l}\text { Blanchard et } \\
\text { al [16] }\end{array}$} & 2020 & 14 & Homo & 823 & $\begin{array}{l}\text { OR of } \\
\text { second- } \\
\text { versus first- } \\
\text { born sons }\end{array}$ & $\begin{array}{l}\text { Primary goal was to assess the performance of the the } \\
\text { OR of second- versus firts-born sons. This OR is } \\
\text { computed by restricting the sampling space to } \\
\text { individuals who reported exactly one brother but any } \\
\text { number of sisters and comparing the ratios of second- } \\
\text { versus first-born sons in homo- versus heterosexual } \\
\text { men. In light of parts I and II below it is easy to see } \\
\text { that, just like the OBOR, this OR fails to account for a } \\
\text { more general excess of older siblings. See } \\
\text { supplementary material for a detailed account of [16]. }\end{array}$ \\
\hline & & & Hetero & 1,885 & & \\
\hline \multirow[t]{2}{*}{$\begin{array}{l}\text { Blanchard et } \\
\text { al. [8] }\end{array}$} & 2020 & 24 & Homo & 5,963 & OBOR & $\begin{array}{l}\text { The primary stated goals of this meta-analysis were } \\
\text { (1) "to examine the evidence for the FBOE in } \\
\text { pedophiles", (2) to compare its strength to that of the } \\
\text { FBOE in individuals attracted to mature adults } \\
\text { ("Teleiophiles"), and (3) to determine if an excess of } \\
\text { older sisters could be detected in these two groups. }\end{array}$ \\
\hline & & & Hetero & 12,250 & & \\
\hline
\end{tabular}

Notes. Year = year of publication, \# samples = number of samples included in the meta-analysis, group = sexual orientation (homo = homosexual, hetero = heterosexual), $\mathrm{N}=$ number of participants, "-" = not reported, $O B O R=$ older brothers odds ratio, $O R=$ odds ratio. 
Table 2. Equations of currently recommended measures for quantifying the excess of older brothers in homosexual men.

\begin{tabular}{|c|c|c|}
\hline Introduced by & Measure & Equation \\
\hline Blanchard [5] & Older brothers odds ratio & $O B O R=\frac{\# \mathrm{OB}_{\mathrm{Hom}} / \# \mathrm{Other}_{\mathrm{Hon}}}{\# \mathrm{OB}_{\mathrm{Het}} / \# \mathrm{Other}_{\mathrm{Het}}}$ \\
\hline Blanchard [17] & Older sisters odds ratio & OSOR $=\frac{\# \mathrm{OS}_{\mathrm{Hom}} / \# \mathrm{Other}_{\mathrm{Hom}}}{\# \mathrm{OS}_{\mathrm{Het}} / \# \mathrm{Other}_{\mathrm{Het}}}$ \\
\hline Blanchard [19] & Modified ratio of older brothers & 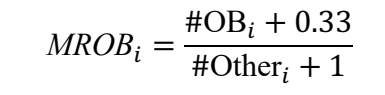 \\
\hline & Modified ratio of older sisters & $\operatorname{MROS}_{i}=\frac{\mathrm{\# OS}_{i}+0.33}{\text { \#ther }_{i}+1}$ \\
\hline & Modified proportion of older brothers & $M P O B_{i}=\frac{\# \mathrm{OB}_{i}+0.25}{\# \mathrm{All}_{i}+1}$ \\
\hline & Modified proportion of older sisters & $M P O S_{i}=\frac{\# \mathrm{OS}_{i}+0.25}{\# \mathrm{All}_{i}+1}$ \\
\hline
\end{tabular}

Notes. $\# \mathrm{OB}=$ Number of older brothers, \#OS = Number of older sisters, $\# \mathrm{YB}=$ Number of younger brothers, \#YS = Number of younger sisters. 
Table 3. Correlation matrix used to simulate participants.

\begin{tabular}{lllll} 
& \#OB & \#OS & \#YB & \#YS \\
\hline \#OB & 1 & & & \\
\#OS & .158 & 1 & & \\
\#YB & -.092 & -.071 & 1 & \\
\#YS & -.057 & -.066 & .063 & 1
\end{tabular}

Notes. $\# \mathrm{OB}=$ Number of older brothers, \#OS = Number of older sisters, \# $\mathrm{YB}=$ Number of younger brothers, \#YS = Number of younger sisters. 
Table 4. Regression models fitted to simulated data.

\begin{tabular}{lll}
\hline Model & Equation & Description \\
\hline Models warned against by [19] & \\
Model 1 & $\operatorname{logit}\left[\mathrm{P}\left(\mathrm{Hom}_{i}\right)\right]=\beta_{0}+\beta_{\# \mathrm{OB}} \# \mathrm{OB}_{i}+\beta_{\# \mathrm{OS}} \# \mathrm{OS}_{i}$ & $\begin{array}{l}\text { Predicts the logit of the probability of } \\
\text { homosexual orientation from the number of } \\
\text { older brothers and older sisters. Effect of } \\
\text { interest: } \beta_{\# \mathrm{OB}} .\end{array}$
\end{tabular}

Models recommended by $[5,8,18,19]$

Model $2 \operatorname{logit}\left[\mathrm{P}\left(\operatorname{Hom}_{i}\right)\right]=\beta_{0}+\beta_{M R O B} M R O B_{i}+\beta_{M R O S} M R O S_{i}$

Predicts the logit of the probability of homosexual orientation from the modified ratio of older brothers $(M R O B)$ and modified ratio of older sisters (MROS). Effect of interest: $\beta_{\text {MROB }}$.

Model 3

$\operatorname{logit}\left[\mathrm{P}\left(\mathrm{Hom}_{i}\right)\right]=\beta_{0}+\beta_{M P O B} M P O B_{i}+\beta_{M P O S} M P O S_{i}$

Model 4

$\operatorname{logit}\left[\mathrm{P}\left(\mathrm{OB}_{i j}\right)\right]=\beta_{0}+\beta_{\mathrm{Hom}} \# \mathrm{Hom}_{i}$

Model 5

$\operatorname{logit}\left[\mathrm{P}\left(\mathrm{OS}_{i j}\right)\right]=\beta_{0}+\beta_{\mathrm{Hom}} \# \mathrm{Hom}_{i}$

Models implied by [19]

Model $6 \quad \operatorname{logit}\left[\mathrm{P}\left(\operatorname{Hom}_{i}\right)\right]=\beta_{0}+\beta_{\# \mathrm{OB}} \# \mathrm{OB}_{i}+\beta_{\# \mathrm{All}} \# \mathrm{All}_{i}$

Model 7

$\operatorname{logit}\left[\mathrm{P}\left(\operatorname{Hom}_{i}\right)\right]=\beta_{0}+\beta_{\# \mathrm{OB}} \# \mathrm{OB}_{i}+\beta_{\# \text { Other }} \# \mathrm{Other}_{i}$

Predicts the logit of the probability of homosexual orientation from the MPOB and MPOS. Effect of interest: $\beta_{\mathrm{MROB}}$.

Predicts the logit of the probability of observing an older brother among all siblings. Effect of interest: $\ln O B O R=\beta_{\mathrm{Hom}}$.

Predicts the logit of the probability of observing an older sister among all siblings. Effect of interest: $\ln O S O R=\beta_{\mathrm{Hom}}$.

Predicts the logit of the probability of homosexual orientation from the number of older brothers and the number of all siblings. Effect of interest: $\beta_{\# \mathrm{OB}}$.

Predicts the logit of the probability of homosexual orientation from the number of older brothers and the number of all other siblings (i.e., siblings who are not older brothers). Effect of interest: $\beta_{\# \mathrm{OB}}$.

Models controlling for the number of older siblings

$\begin{array}{ll}\text { Model } 8 & \operatorname{logit}\left[\mathrm{P}\left(\mathrm{Hom}_{i}\right)\right] \\ & =\beta_{0}+\beta_{\# \mathrm{OB}-\# \mathrm{OS}}\left(\# \mathrm{OB}_{i}-\# \mathrm{OS}_{i}\right)+\beta_{\# \mathrm{Older}} \# \mathrm{Older}_{i}\end{array}$

Model 9

$\operatorname{logit}\left[\mathrm{P}\left(\operatorname{Hom}_{i}\right)\right]=\beta_{0}+\beta_{\# \mathrm{OB}} \# \mathrm{OB}_{i}+\beta_{\# \mathrm{Older}} \# \mathrm{Older}_{i}$

Model 10

$\operatorname{logit}\left[\mathrm{P}\left(\mathrm{OB}_{i j}\right)\right]=\beta_{0}+\beta_{\mathrm{Hom}} \# \mathrm{Hom}_{i}$
Predicts the logit of the probability of homosexual orientation from the difference of the number of older brothers and older sisters and the sum of older brothers and

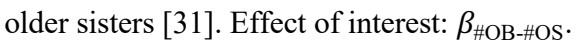

Predicts the logit of the probability of homosexual orientation from the the number of older brothers and the sum of older brothers and older sisters. Effect of interest:

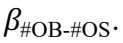

Predicts the logit of the probability of observing an older brother among older siblings (i.e., the sampling space is restricted to older siblings). Effect of interest: $\ln O R=$ $\beta_{\text {Hom }}$.

Notes. The subscripts $i$ and $j$ index the participants and reported siblings, respectively. \#OB, \#OS, \#Older, \#All, and \#Other refer to the number of older brothers, the number of older sisters, the number of older siblings, the number of all siblings and the number of other siblings (i.e, siblings who are not older 
brothers), respectively. Orientation is a binary variable with values $0=$ heterosexual and $1=$ homosexual. $\mathrm{OB}_{i j}, \mathrm{OS}_{i j}$ and $\mathrm{Older}_{i j}$ are binary variables with values 0 and 1 , indicating the absence vs. presence of the event that the th sibling is an older brother, an older sister, or an older sibling of the participant $i$ who reported him or her. MROB, MROS, MPOB and MPOS refer to the modified ratio of older brothers, the modified ratio of older sisters, the modified proportion of older brothers and the modified proportion of older sisters, respectively. 
Table 5. Means and standard deviations of older brothers and sisters for the full set of male samples.

\begin{tabular}{|c|c|c|c|c|c|c|c|c|c|c|c|c|}
\hline Sample & Year & $\begin{array}{l}\text { Orientatio } \\
\mathrm{n}\end{array}$ & $\mathrm{N}$ & $\begin{array}{l}\text { Mean Number of } \\
\text { Older Brothers } \\
\text { (SD) }\end{array}$ & $\begin{array}{l}\text { Mean Number } \\
\text { of Older } \\
\text { Sisters (SD) }\end{array}$ & 1 & 2 & 3 & 4 & 5 & 6 & 7 \\
\hline \multirow{2}{*}{$\begin{array}{l}\text { Blanchard and Sheridan } \\
\text { [48] }\end{array}$} & \multirow[t]{2}{*}{1992} & Homo & 193 & $1.04(-)$ & $0.82(-)$ & \multirow[t]{2}{*}{$\mathrm{X}$} & \multirow{2}{*}{\multicolumn{2}{|c|}{$\mathrm{X}$}} & \multirow{2}{*}{\multicolumn{4}{|c|}{$\mathrm{X}$}} \\
\hline & & Hetero & 273 & $0.49(-)$ & $0.48(-)$ & & & & & & & \\
\hline \multirow{2}{*}{$\begin{array}{l}\text { Blanchard and Zucker } \\
\text { [49] }\end{array}$} & \multirow[t]{2}{*}{1994} & Homo & 569 & $0.50(-)$ & $0.45(-)$ & \multirow[t]{2}{*}{$\mathrm{X}$} & \multirow{2}{*}{\multicolumn{2}{|c|}{$\mathrm{X}$}} & \multirow{2}{*}{\multicolumn{2}{|c|}{$\mathrm{X}$}} & & \multirow[t]{2}{*}{$\mathrm{X}$} \\
\hline & & Hetero & 281 & $0.44(-)$ & $0.36(-)$ & & & & & & & \\
\hline \multirow{2}{*}{ Zucker and Blanchard } & \multirow[t]{2}{*}{1994} & Homo & 98 & $0.45(-)$ & $0.49(-)$ & \multirow[t]{2}{*}{$\mathrm{X}$} & $\mathrm{X}$ & & $\mathrm{X}$ & & & $\mathrm{X}$ \\
\hline & & Hetero & 84 & $0.39(-)$ & $0.35(-)$ & & & & & & & \\
\hline Blanchard et al. [51] & 1995 & Homo & 156 & $0.63(-)$ & $0.43(-)$ & $\mathrm{X}$ & $\mathrm{X}$ & & $\mathrm{X}$ & & & \\
\hline & & Hetero & 156 & $0.42(-)$ & $0.39(-)$ & & & & & & & \\
\hline Blanchard and Bogaert & 1996 & Homo & 799 & $0.70(1.12)$ & $0.59(0.97)$ & $\mathrm{X}$ & $\mathrm{X}$ & & $\mathrm{X}$ & & & $\mathrm{X}$ \\
\hline$[2]$ & & Hetero & 3807 & $0.58(0.98)$ & $0.54(0.93)$ & & & & & & & \\
\hline Blanchard and Bogaert & 1996 & Homo & 302 & $0.71(-)$ & $0.60(-)$ & $X$ & $\mathrm{X}$ & & $\mathrm{X}$ & & & $\mathrm{X}$ \\
\hline [1] & & Hetero & 302 & $0.69(-)$ & $0.68(-)$ & & & & & & & \\
\hline Blanchard et al. [43] & 1996 & Homo & 83 & $1.04(1.19)$ & $0.73(1.04)$ & $\mathrm{X}$ & $\mathrm{X}$ & & $\mathrm{X}$ & & & \\
\hline Study 1 & & Hetero & 58 & $0.76(1.41)$ & $0.52(0.75)$ & & & & & & & \\
\hline Blanchard et al. [43] & 1996 & Homo & 21 & $0.81(0.68)$ & $0.24(0.44)$ & $\mathrm{X}$ & $\mathrm{X}$ & & $\mathrm{X}$ & & & \\
\hline Study 2 & & Hetero & 21 & $0.33(0.58)$ & $0.05(0.22)$ & & & & & & & \\
\hline Bogaert et al. [52] & 1997 & Homo & 68 & $0.76(-)$ & $0.81(-)$ & & $\mathrm{X}$ & & $\mathrm{X}$ & & & $\mathrm{X}$ \\
\hline & & Hetero & 57 & $0.56(-)$ & $0.70(-)$ & & & & & & & \\
\hline $\begin{array}{l}\text { Blanchard and Bogaert } \\
\text { [53] }\end{array}$ & 1998 & Homo & 157 & $0.82(-)$ & $0.71(-)$ & $\mathrm{X}$ & $\mathrm{X}$ & & $X$ & & $\mathrm{X}$ & $\mathrm{X}$ \\
\hline Offenders against adults & & Hetero & 173 & $0.89(-)$ & $0.91(-)$ & & & & & & & \\
\hline $\begin{array}{l}\text { Blanchard and Bogaert } \\
\text { [53] }\end{array}$ & 1998 & Homo & 42 & $1.00(-)$ & $0.95(-)$ & & $\mathrm{X}$ & & $\mathrm{X}$ & & $\mathrm{X}$ & $\mathrm{X}$ \\
\hline $\begin{array}{l}\text { Offenders against } \\
\text { children }\end{array}$ & & Hetero & 143 & $1.08(-)$ & $1.09(-)$ & & & & & & & \\
\hline Blanchard and Bogaert & 1998 & Homo & 69 & $1.14(-)$ & $1.13(-)$ & & $\mathrm{X}$ & & $\mathrm{X}$ & & $\mathrm{X}$ & $\mathrm{X}$ \\
\hline $\begin{array}{l}\text { Offenders against } \\
\text { pubescents }\end{array}$ & & Hetero & 127 & $1.17(-)$ & $0.94(-)$ & & & & & & & \\
\hline Blanchard et al. [106] & 1998 & Homo & 385 & $0.53(0.90)$ & $0.43(0.77)$ & $\mathrm{X}$ & $\mathrm{X}$ & & $\mathrm{X}$ & & $\mathrm{X}$ & $\mathrm{X}$ \\
\hline & & Hetero & 225 & $0.32(0.65)$ & $0.43(0.85)$ & & & & & & & \\
\hline Blanchard et al. [75] & 2000 & Homo & 65 & $1.08(-)$ & $0.78(-)$ & & $\mathrm{X}$ & & $\mathrm{X}$ & & & \\
\hline & & Hetero & 152 & $0.76(-)$ & $0.69(-)$ & & & & & & & \\
\hline Green [60] & 2000 & Homo & 106 & $0.90(1.11)$ & $0.79(1.11)$ & & & $\mathrm{X}$ & $\mathrm{X}$ & & & \\
\hline & & Hetero & 135 & $0.58(0.88)$ & $0.61(0.95)$ & & & & & & & \\
\hline Ellis and Blanchard [54] & 2001 & Homo & 175 & $0.67(-)$ & $0.49(-)$ & & $\mathrm{X}$ & & $\mathrm{X}$ & & $\mathrm{X}$ & $\mathrm{X}$ \\
\hline & & Hetero & 971 & $0.51(-)$ & $0.50(-)$ & & & & & & & \\
\hline Rahman et al. [107] & 2004 & Homo & 60 & $0.58(0.74)$ & $0.85(1.32)$ & & & $\mathrm{X}$ & & & & \\
\hline & & Hetero & 60 & $0.48(0.79)$ & $0.53(0.85)$ & & & & & & & \\
\hline Bogaert [63] & 2005 & Homo & 79 & $0.91(-)$ & $0.63(-)$ & & & & & $\mathrm{X}$ & & \\
\hline & & Hetero & 2721 & $0.69(-)$ & $0.65(-)$ & & & & & & & \\
\hline King et al. [108] & 2005 & Homo & 301 & $0.66(0.88)$ & $0.59(0.88)$ & & & $\mathrm{X}$ & $\mathrm{X}$ & & & $\mathrm{X}$ \\
\hline & & Hetero & 404 & $0.47(0.71)$ & $0.43(0.73)$ & & & & & & & \\
\hline Rahman [109] & 2005 & Homo & 30 & $1.09(0.90)$ & $0.58(0.99)$ & & & $\mathrm{X}$ & & & & \\
\hline & & Hetero & 31 & $0.40(0.72)$ & $0.53(0.97)$ & & & & & & & \\
\hline Blanchard et al. [55] & 2006 & Homo & 92 & $1.07(-)$ & $0.86(-)$ & & & & $\mathrm{X}$ & & & \\
\hline "Blanchard" subsample & & Hetero & 672 & $0.83(-)$ & $0.82(-)$ & & & & & & & \\
\hline Blanchard et al. [55] & 2006 & Homo & 280 & $0.50(-)$ & $0.41(-)$ & & & & $\mathrm{X}$ & & $\mathrm{X}$ & $\mathrm{X}$ \\
\hline $\begin{array}{l}\text { "Bogaert other"" } \\
\text { subsample }\end{array}$ & & Hetero & 222 & $0.38(-)$ & $0.41(-)$ & & & & & & & \\
\hline Blanchard et al. [55] & 2006 & Homo & 267 & $0.82(-)$ & $0.65(-)$ & & & & $\mathrm{X}$ & & $\mathrm{X}$ & $\mathrm{X}$ \\
\hline $\begin{array}{l}\text { "Bogaert non-biological } \\
\text { family" subsample }\end{array}$ & & Hetero & 148 & $0.51(-)$ & $0.45(-)$ & & & & & & & \\
\hline Frisch and Hviid [35] & 2006 & Homo & 1890 & $0.37(0.62)$ & $0.30(0.59)$ & & & & & $\mathrm{X}$ & & \\
\hline & & Hetero & 429181 & $0.34(0.61)$ & $0.27(0.55)$ & & & & & & & \\
\hline Blanchard and Lippa & 2007 & Homo & 8279 & $0.53(-)$ & $0.51(-)$ & & & & & & & \\
\hline$[62]$ & & Hetero & 79519 & $0.45(-)$ & $0.44(-)$ & & & & & & & \\
\hline Vasey and VanderLaan & 2007 & Homo & 83 & $2.27(1.84)$ & $2.08(1.71)$ & & & & $\mathrm{X}$ & & & \\
\hline [71] & & Hetero & 114 & $1.23(1.37)$ & $1.25(1.20)$ & & & & & & & \\
\hline Zucker et al. [110] & 2007 & Homo & 43 & $0.26(0.49)$ & $0.84(1.13)$ & & & & & & & \\
\hline & & Hetero & 49 & $0.43(0.65)$ & $1.00(1.29)$ & & & & & & & \\
\hline Rahman et al. [111] & 2008 & Homo & 20 & $0.60(0.82)$ & $0.45(0.60)$ & & & $\mathrm{X}$ & & & & \\
\hline Non-white subsample & & Hetero & 53 & $0.81(1.05)$ & $0.84(1.13)$ & & & & & & & \\
\hline Rahman et al. [111] & 2008 & Homo & 127 & $0.55(0.82)$ & $0.49(0.79)$ & & & $\mathrm{X}$ & & & & \\
\hline
\end{tabular}




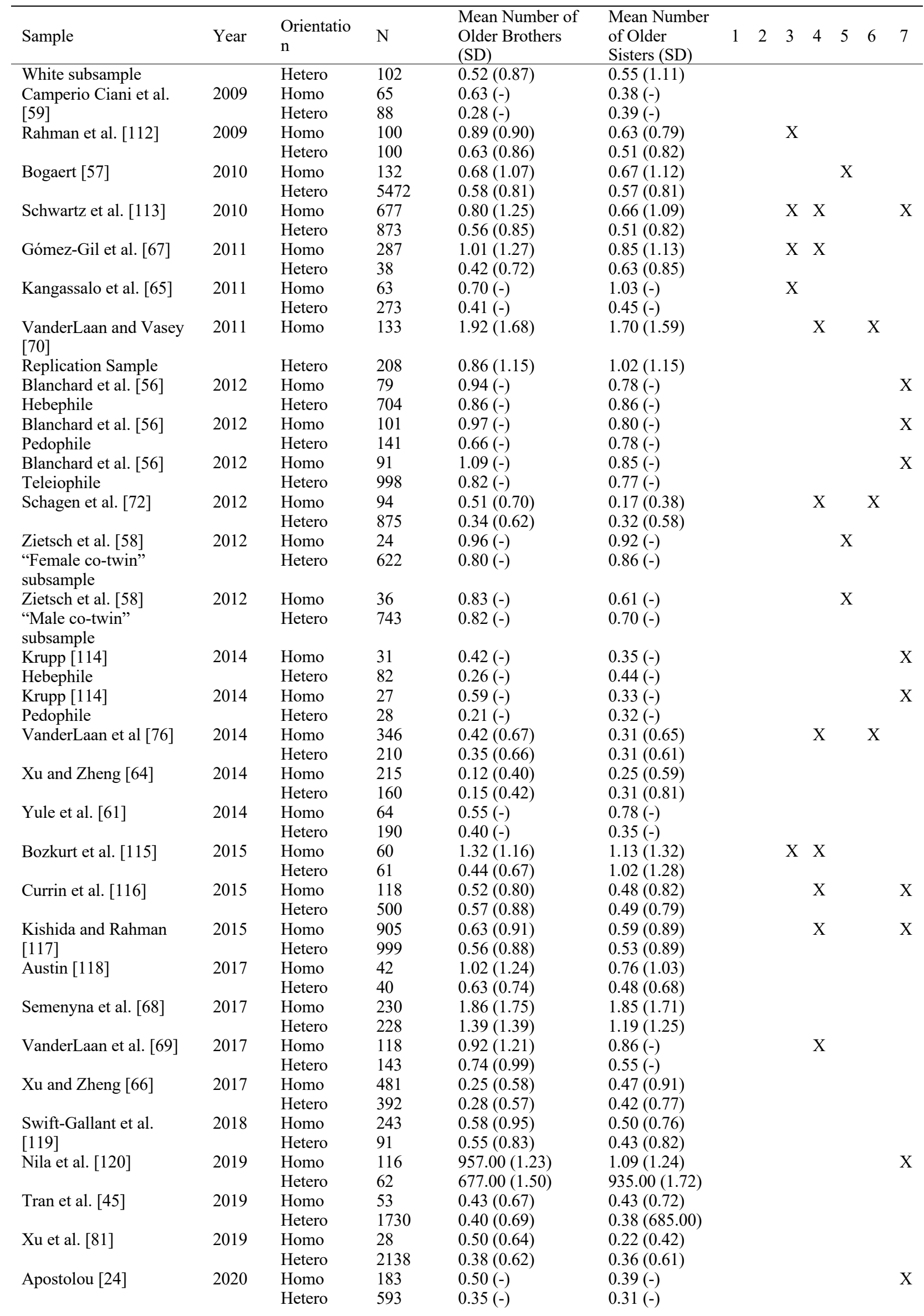




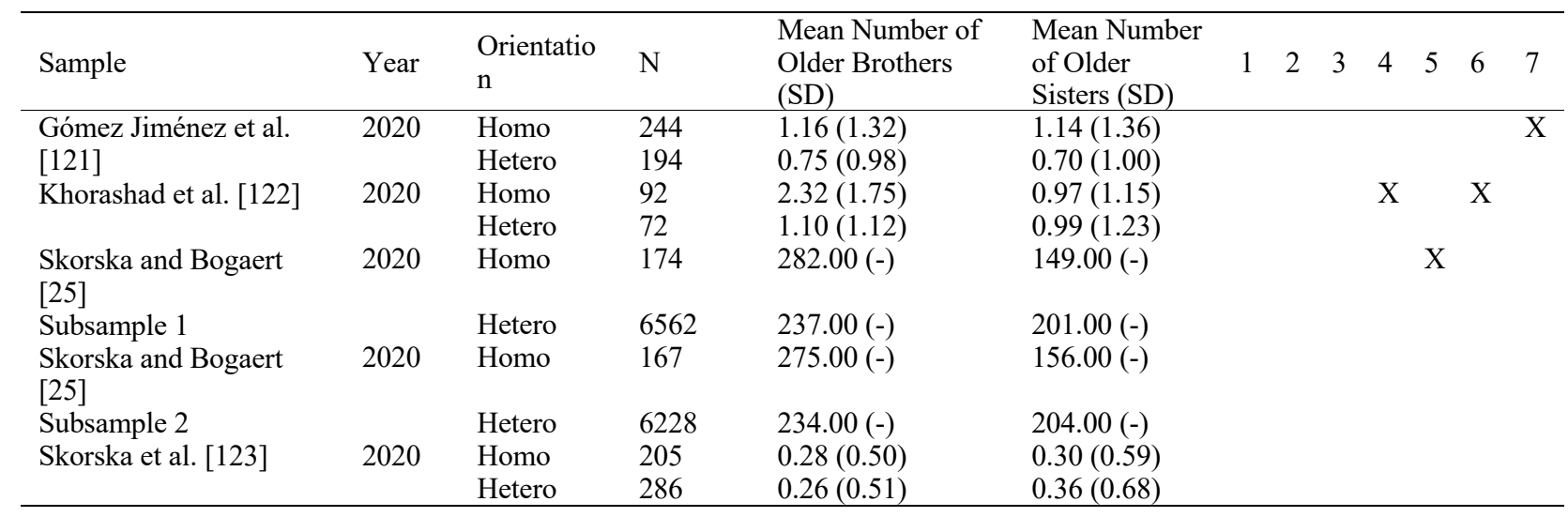

Notes. Column "Group" denotes sexual orientation $($ Homo = homosexual, Hetero $=$ heterosexual) of the sample. "-" denots non-reported (missing) values. Cell entries of X (vs. blank space) in the rightmost column indicate whether a given sample was included (vs. not) in each of the seven previous metaanalyses. The columns labelled " 1 " through "7" correspond to the meta-analyses by [14] (=1), [7] (=2), [15] $(=3),[5](=4),[13](=5),[16](=6),[8](=7)$. 
Table 6. Means and standard deviations of older brothers and sisters for the full set of female samples.

\begin{tabular}{|c|c|c|c|c|c|}
\hline Sample & Year & Orientation & $\mathrm{N}$ & $\begin{array}{l}\text { Mean Number of Older Brothers } \\
\text { (SD) }\end{array}$ & $\begin{array}{l}\text { Mean Number of Older Sisters } \\
\text { (SD) }\end{array}$ \\
\hline \multirow[t]{2}{*}{ Blanchard et al. [106] } & 1998 & Homo & 162 & $0.41(0.75)$ & $0.30(0.69)$ \\
\hline & & Hetero & 192 & $0.43(0.83)$ & $0.41(0.81)$ \\
\hline \multirow[t]{2}{*}{ Green $[60]$} & 2000 & Homo & 73 & $0.58(1.05)$ & $0.74(1.09)$ \\
\hline & & Hetero & 7 & $0.71(0.95)$ & $0.14(0.38)$ \\
\hline \multirow[t]{2}{*}{ Rahman et al. [107] } & 2004 & Homo & 60 & $0.63(0.97)$ & $0.68(1.09)$ \\
\hline & & Hetero & 60 & $0.65(1.05)$ & $0.45(1.04)$ \\
\hline \multirow[t]{2}{*}{ Rahman [109] } & 2005 & Homo & 30 & $0.65(0.89)$ & $0.58(0.90)$ \\
\hline & & Hetero & 29 & $0.63(0.76)$ & $1.13(1.59)$ \\
\hline \multirow[t]{2}{*}{ Frisch and Hviid [35] } & 2006 & Homo & 1537 & $0.40(-)$ & $0.28(-)$ \\
\hline & & Hetero & 453121 & $0.38(-)$ & $0.31(-)$ \\
\hline \multirow{4}{*}{$\begin{array}{l}\text { Blanchard and Lippa } \\
\text { [62] } \\
\text { Bogaert [57] }\end{array}$} & 2007 & Homo & 7013 & $0.43(-)$ & $0.37(-)$ \\
\hline & & Hetero & 64968 & $0.48(-)$ & $0.43(-)$ \\
\hline & 2010 & Homo & 75 & $0.55(0.83)$ & $0.71(0.99)$ \\
\hline & & Hetero & 5335 & $0.59(0.79)$ & $0.56(0.80)$ \\
\hline \multirow[t]{2}{*}{ Gómez-Gil et al. [67] } & 2011 & Homo & 154 & $0.82(1.02)$ & $0.79(1.14)$ \\
\hline & & Hetero & 6 & $1.50(1.98)$ & $1.50(2.07)$ \\
\hline \multirow[t]{2}{*}{ Schagen et al. [72] } & 2012 & Homo & 95 & $0.25(-)$ & $0.23(-)$ \\
\hline & & Hetero & 914 & $0.36(-)$ & $0.33(-)$ \\
\hline \multirow[t]{2}{*}{$\mathrm{Xu}$ and Zheng [64] } & 2014 & Homo & 42 & $0.10(0.07)$ & $0.07(-)$ \\
\hline & & Hetero & 255 & $0.15(0.45)$ & $0.19(0.47)$ \\
\hline \multirow[t]{2}{*}{$\mathrm{Xu}$ and Zheng [66] } & 2017 & Homo & 431 & $0.16(0.45)$ & $0.21(0.70)$ \\
\hline & & Hetero & 554 & $0.22(0.57)$ & $0.23(0.51)$ \\
\hline \multirow{2}{*}{ Tran et al. [45] } & 2019 & Homo & 28 & $0.32(0.62)$ & $0.46(0.84)$ \\
\hline & & Hetero & 1947 & $0.36(0.66)$ & $0.38(0.67)$ \\
\hline \multirow{2}{*}{$\mathrm{Xu}$ et al. [81] } & 2019 & Homo & 16 & $0.63(0.81)$ & $0.31(0.60)$ \\
\hline & & Hetero & 2391 & $0.38(0.63)$ & $0.37(0.60)$ \\
\hline \multirow[t]{2}{*}{ Apostolou [24] } & 2020 & Homo & 76 & $0.41(-)$ & $0.24(-)$ \\
\hline & & Hetero & 366 & $0.36(-)$ & $0.34(-)$ \\
\hline \multirow[t]{2}{*}{ Khorashad et al. [122] } & 2020 & Homo & 107 & $1.04(1.26)$ & $1.29(1.36)$ \\
\hline & & Hetero & 78 & $1.15(1.27)$ & $1.05(1.27)$ \\
\hline $\begin{array}{l}\text { Skorska and Bogaert } \\
{[25]}\end{array}$ & 2020 & Homo & 143 & $0.20(-)$ & $0.20(-)$ \\
\hline Subsample 1 & & Hetero & 7727 & $0.22(-)$ & $0.21(-)$ \\
\hline $\begin{array}{l}\text { Skorska and Bogaert } \\
{[25]}\end{array}$ & 2020 & Homo & 136 & $0.21(-)$ & $0.20(-)$ \\
\hline Subsample 2 & & Hetero & 7297 & $0.23(-)$ & $0.21(-)$ \\
\hline
\end{tabular}

Notes. The table column "Group" denotes sexual orientation (Homo $=$ homosexual, Hetero $=$ heterosexual) of sample. "-" denotes non-reported missing values. None of these female samples has been included in any of the seven prior related meta-analyses 
Table 7. Specification matrix indicating the factor-level of each of the 64 male samples for each of the 10 Which factors.

\begin{tabular}{|c|c|c|c|c|c|c|c|c|c|c|}
\hline Sample & $\begin{array}{l}\text { In } \\
\text { Previous } \\
\text { MA }\end{array}$ & $\mathrm{Lab}$ & Archives & Feminine & GDY & Clinical & Classification & Probability & Stopping & $\begin{array}{l}\text { Teleio- vs. Pedo- } \\
\text { /Hebephiles }\end{array}$ \\
\hline Blanchard and Sheridan [48] & $\mathrm{X}$ & $\mathrm{X}$ & & $\mathrm{X}$ & $\mathrm{X}$ & $\mathrm{X}$ & Indirect & & & \\
\hline Blanchard and Zucker [49] & $\mathrm{X}$ & $\mathrm{X}$ & & & & & Various & & & \\
\hline Zucker and Blanchard [50] & $\mathrm{X}$ & $\mathrm{X}$ & $\mathrm{X}$ & & & & Various & & & \\
\hline Blanchard et al. [51] & $\mathrm{X}$ & $\mathrm{X}$ & & $\mathrm{X}$ & $\mathrm{X}$ & $\mathrm{X}$ & Indirect & & & \\
\hline Blanchard and Bogaert [2] & $\mathrm{X}$ & $\mathrm{X}$ & $\mathrm{X}$ & & & & Indirect & & & \\
\hline Blanchard and Bogaert [1] & $\mathrm{X}$ & $\mathrm{X}$ & & & & & Single Item & & & \\
\hline Blanchard et al. [43] Study 1 & $\mathrm{X}$ & $\mathrm{X}$ & $\mathrm{X}$ & $\mathrm{X}$ & $\mathrm{X}$ & $\mathrm{X}$ & Single Item & & & \\
\hline Blanchard et al. [43] Study 2 & $\mathrm{X}$ & $\mathrm{X}$ & $\mathrm{X}$ & $\mathrm{X}$ & $\mathrm{X}$ & $\mathrm{X}$ & None & & & \\
\hline Bogaert et al. [52] & $\mathrm{X}$ & $\mathrm{X}$ & & & & $\mathrm{X}$ & Various & & & $X$ \\
\hline Blanchard and Bogaert [53] & $\mathrm{X}$ & $\mathrm{X}$ & $\mathrm{X}$ & & & $\mathrm{X}$ & Indirect & & & \\
\hline Offenders against adults & & & & & & & & & & \\
\hline Blanchard and Bogaert [53] & $\mathrm{X}$ & $\mathrm{X}$ & $\mathrm{X}$ & & & $\mathrm{X}$ & Indirect & & & $\mathrm{X}$ \\
\hline Offenders against children & & & & & & & & & & \\
\hline $\begin{array}{l}\text { Blanchard and Bogaert [53] } \\
\text { Offenders against pubescents }\end{array}$ & $\mathrm{X}$ & $\mathrm{X}$ & $\mathrm{X}$ & & & $\mathrm{X}$ & Indirect & & & $\mathrm{X}$ \\
\hline Blanchard et al. [106] & $\mathrm{X}$ & $\mathrm{X}$ & & & & & Various & & & \\
\hline Blanchard et al. [75] & $\mathrm{X}$ & $\mathrm{X}$ & $\mathrm{X}$ & & & $\mathrm{X}$ & Various & & & $\mathrm{X}$ \\
\hline Green [60] & $\mathrm{X}$ & & $\mathrm{X}$ & $\mathrm{X}$ & $\mathrm{X}$ & $\mathrm{X}$ & Various & & & \\
\hline Ellis and Blanchard [54] & $\mathrm{X}$ & $\mathrm{X}$ & & & & & Single Item & & & \\
\hline Rahman et al. [107] & $\mathrm{X}$ & & & & & & Various & & & \\
\hline Bogaert [63] & $\mathrm{X}$ & $\mathrm{X}$ & $\mathrm{X}$ & & & & Various & $\mathrm{X}$ & & \\
\hline King et al. [108] & $\mathrm{X}$ & & $\mathrm{X}$ & & & $\mathrm{X}$ & Various & & & \\
\hline Rahman [109] & $\mathrm{X}$ & & & & & & Various & & & \\
\hline $\begin{array}{l}\text { Blanchard et al. [55] } \\
\text { "Blanchard" subsample }\end{array}$ & $\mathrm{X}$ & $\mathrm{X}$ & & & & $\mathrm{X}$ & Various & & & \\
\hline $\begin{array}{l}\text { Blanchard et al. [55] "Bogaert } \\
\text { other" subsample }\end{array}$ & $\mathrm{X}$ & $\mathrm{X}$ & & & & & Various & & & \\
\hline $\begin{array}{l}\text { Blanchard et al. [55] "Bogaert } \\
\text { non-biological family" } \\
\text { subsample }\end{array}$ & $\mathrm{X}$ & $\mathrm{X}$ & & & & & Various & & & \\
\hline Frisch and Hviid [35] & $\mathrm{X}$ & & $\mathrm{X}$ & & & & Single Item & $\mathrm{X}$ & & \\
\hline Blanchard and Lippa [62] & & $\mathrm{X}$ & $\mathrm{X}$ & & & & Various & & & \\
\hline Vasey and VanderLaan [71] & $\mathrm{X}$ & $\mathrm{X}$ & & $\mathrm{X}$ & & & Single Item & & & \\
\hline Zucker et al. [110] & & $\mathrm{X}$ & & & $\mathrm{X}$ & $\mathrm{X}$ & Single Item & & & \\
\hline $\begin{array}{l}\text { Rahman et al. [111] Non- } \\
\text { white subsample }\end{array}$ & $\mathrm{X}$ & & $\mathrm{X}$ & & & & Various & & & \\
\hline $\begin{array}{l}\text { Rahman et al. [111] White } \\
\text { subsample }\end{array}$ & $\mathrm{X}$ & & $\mathrm{X}$ & & & & Various & & & \\
\hline Camperio Ciani et al. [59] & & & & & & & Various & & & \\
\hline Rahman et al. [112] & $\mathrm{X}$ & & & & & & Various & & & \\
\hline
\end{tabular}




\begin{tabular}{|c|c|c|c|c|c|c|c|c|c|c|}
\hline Sample & $\begin{array}{c}\text { In } \\
\text { Previous } \\
\text { MA }\end{array}$ & Lab & Archives & Feminine & GDY & Clinical & Classification & Probability & Stopping & $\begin{array}{l}\text { Teleio- vs. Pedo- } \\
\text { /Hebephiles }\end{array}$ \\
\hline Bogaert [57] & $\mathrm{X}$ & $\mathrm{X}$ & $\mathrm{X}$ & & & & Various & $\mathrm{X}$ & & \\
\hline Schwartz et al. [113] & $\mathrm{X}$ & & $\mathrm{X}$ & & & & Various & & & \\
\hline Gómez-Gil et al. [67] & $\mathrm{X}$ & & $\mathrm{X}$ & $\mathrm{X}$ & $\mathrm{X}$ & $\mathrm{X}$ & Single Item & & & \\
\hline Kangassalo et al. [65] & $X$ & & & & & & Various & & & \\
\hline VanderLaan and Vasey [70] & $\mathrm{X}$ & $\mathrm{X}$ & $\mathrm{X}$ & $\mathrm{X}$ & & & Various & & & \\
\hline Replication Sample & & & & & & & & & & \\
\hline $\begin{array}{l}\text { Blanchard et al. [56] } \\
\text { Hebephile }\end{array}$ & $\mathrm{X}$ & $\mathrm{X}$ & $\mathrm{X}$ & & & $\mathrm{X}$ & Indirect & & & $\mathrm{X}$ \\
\hline $\begin{array}{l}\text { Blanchard et al. [56] } \\
\text { Pedophile }\end{array}$ & $\mathrm{X}$ & $\mathrm{X}$ & $\mathrm{X}$ & & & $\mathrm{X}$ & Indirect & & & $\mathrm{X}$ \\
\hline $\begin{array}{l}\text { Blanchard et al. [56] } \\
\text { Teleiophile }\end{array}$ & $\mathrm{X}$ & $\mathrm{X}$ & $\mathrm{X}$ & & & $\mathrm{X}$ & Indirect & & & $\mathrm{X}$ \\
\hline Schagen et al. [72] & $\mathrm{X}$ & $\mathrm{X}$ & $\mathrm{X}$ & $\mathrm{X}$ & $\mathrm{X}$ & $\mathrm{X}$ & None & & & \\
\hline $\begin{array}{l}\text { Zietsch et al. [58] "Female } \\
\text { co-twin" subsample }\end{array}$ & $\mathrm{X}$ & & & & & & Single Item & $X$ & & \\
\hline $\begin{array}{l}\text { Zietsch et al. [58] "Male co- } \\
\text { twin" subsample }\end{array}$ & $\mathrm{X}$ & & & & & & Single Item & $\mathrm{X}$ & & \\
\hline Krupp [114] Hebephile & $\mathrm{X}$ & & & & & $\mathrm{X}$ & - & & & $\mathrm{X}$ \\
\hline Krupp [114] Pedophile & $\mathrm{X}$ & & & & & $\mathrm{X}$ & - & & & $\mathrm{X}$ \\
\hline VanderLaan et al [76] & $\mathrm{X}$ & $\mathrm{X}$ & & $\mathrm{X}$ & $\mathrm{X}$ & $\mathrm{X}$ & Various & & & \\
\hline $\mathrm{Xu}$ and Zheng [64] & & & & & & & Single Item & & & \\
\hline Yule et al. [61] & & & $\mathrm{X}$ & & & & Single Item & & & \\
\hline Bozkurt et al. [115] & $\mathrm{X}$ & & $\mathrm{X}$ & $\mathrm{X}$ & $\mathrm{X}$ & & Single Item & & & \\
\hline Currin et al. [116] & $\mathrm{X}$ & & & & & & Various & & & \\
\hline Kishida and Rahman [117] & $\mathrm{X}$ & & $\mathrm{X}$ & & & & Various & & & \\
\hline Austin [118] & & & & & & & Various & & & \\
\hline Semenyna et al. [68] & & $\mathrm{X}$ & & & & & Various & & & \\
\hline VanderLaan et al. [69] & $\mathrm{X}$ & $\mathrm{X}$ & & $\mathrm{X}$ & $\mathrm{X}$ & $\mathrm{X}$ & Indirect & & & \\
\hline $\mathrm{Xu}$ and Zheng [66] & & & & & & & Various & & & \\
\hline Swift-Gallant et al. [119] & & $\mathrm{X}$ & $\mathrm{X}$ & & & & Various & & & \\
\hline Nila et al. [120] & $\mathrm{X}$ & & & & & & Various & & & \\
\hline Tran et al. [45] & & & $\mathrm{X}$ & & & & Single Item & & & \\
\hline $\mathrm{Xu}$ et al. [81] & & & & & & & Various & $\mathrm{X}$ & & \\
\hline Apostolou [24] & $\mathrm{X}$ & & $\mathrm{X}$ & & & & Various & & & \\
\hline Gómez Jiménez et al. [121] & $\mathrm{X}$ & $\mathrm{X}$ & & & & & Various & & & \\
\hline Khorashad et al. [122] & $X$ & $\mathrm{X}$ & & $\mathrm{X}$ & $\mathrm{X}$ & $\mathrm{X}$ & Indirect & & & \\
\hline $\begin{array}{l}\text { Skorska and Bogaert [25] } \\
\text { Subsample } 1\end{array}$ & $\mathrm{X}$ & $\mathrm{X}$ & $\mathrm{X}$ & & & & Single Item & $\mathrm{X}$ & & \\
\hline Skorska and Bogaert [25] & & $X$ & $\mathrm{X}$ & & & & Single Item & $\mathrm{X}$ & & \\
\hline Subsample 2 & & & & & & & & & & \\
\hline Skorska et al. [123] & & $\mathrm{X}$ & $\mathrm{X}$ & & & & Various & & & \\
\hline
\end{tabular}


Note. Studies are ordered by year of publication. "-" = not reported (missing). For the nine binary specification factors, the table entries ("X" vs. blank cell) correspond to: the sample was included in any of the seven previous meta-analyses vs. not (for the factor In Previous MA); sample was recruited in a clinical setting vs. not (for the factor Clinical); sample was denoted as "feminine" by [4] vs. not (for the factor Feminine); sample contained participants with GDY diagnosis vs. not (for the factor GDY); sample was obtained using a random sampling procedure vs. not (for the factor Probability); sample contained in a publication with Blanchard, Bogaert, Zucker, or VanderLaan among the authors vs. not (for the factor Lab); sample was declard as consisting of "pedo- or hebephiles" vs.

"teleiophiles" by [16] (for the factor Pedo-/Hebephiles); a stopping rule was declared as present vs. not (for the factor Stopping); and sample contained in a study publised in the journal Archives of Sexual Behavior vs. not (for the factor Archives). For the factor Classification four levels indicating how sexual orientation was assessed: Level "Various" indicates a mixture of methods or questionnaire summary scores; level "Indirect" indicates that behavioural measures or expert ratings were used; level "Single Item" indicates that only a single piece of information such as self-identified sexual orientation was used; level "none" indicates that sexual orientation was not assessed at all. 
Table 8. Summary of results of fixed- and random-effects for the re-analyses of [5], [17] and [8], as well as the meta-analyses for the two sets consisting of all samples of men and women.

\begin{tabular}{|c|c|c|c|c|c|c|c|c|c|c|}
\hline Set & $\mathrm{k}$ & Group & $\mathrm{N}$ & \#OB & \#OS & $\begin{array}{l}\text { Effect } \\
\text { Size (in } \\
\text { ln units) }\end{array}$ & $\mathrm{FEM}[C I]$ & $\operatorname{REM}[C I]$ & $\hat{\tau}[C I]$ & $P I$ \\
\hline \multirow{3}{*}{$\begin{array}{l}\text { Men } \\
\text { included } \\
\text { in [5] } \\
\text { Men }\end{array}$} & \multirow[b]{2}{*}{31} & Homo & 7,141 & 5,447 & 4,523 & OR & $0.14[0.08,0.20]$ & $0.16[0.09,0.24]$ & $0.12[0.00,0.25]$ & {$[-0.09,0.41]$} \\
\hline & & Hetero & 12,504 & 7,245 & 6,885 & OBOR & $0.32[0.28,0.37]$ & $0.39[0.28,0.51]$ & $0.27[0.18,0.39]$ & {$[-0.14,0.93]$} \\
\hline & \multirow[b]{2}{*}{6} & Homo & 2,335 & 971 & 778 & $O R$ & $0.03[-0.07,0.12]$ & $0.12[-0.06,0.30]$ & $0.14[0.00,0.46]$ & {$[-0.22,0.45]$} \\
\hline $\begin{array}{l}\text { included } \\
\text { in [13] }\end{array}$ & & Hetero & 445,301 & 155,579 & 123,289 & OBOR & $0.20[0.12,0.27]$ & $0.20[0.12,0.27]$ & $0.00[0.00,0.29]$ & - \\
\hline Men & \multirow[b]{2}{*}{24} & Homo & 6,084 & 4,241 & 3,712 & $O R$ & $0.10[0.04,0.16]$ & $0.10[0.04,0.16]$ & $0.00[0.00,0.12]$ & - \\
\hline $\begin{array}{l}\text { included } \\
\text { in [8] }\end{array}$ & & Hetero & 12,118 & 7,303 & 6,933 & $O B O R$ & $0.25[0.20,0.30]$ & $0.25[0.20,0.30]$ & $0.00[0.00,0.13]$ & \\
\hline Men & 64 & Homo & $\begin{array}{l}20,863 \\
552365\end{array}$ & 12,863 & 11,610 & $O R$ & $0.06[0.03,0.09]$ & $0.12[0.07,0.18]$ & $0.13[0.05,0.21]$ & {$[-0.13,0.38]$} \\
\hline Women & \multirow{2}{*}{17} & Homo & 10,178 & 4271 & 3,676 & $O R$ & $0.07[0.02,0.12]$ & $0.07[-0.12,0.26]$ & $\begin{array}{l}0.20[0.14,0.28] \\
0.28[0.02,0.52]\end{array}$ & {$[-0.51,0.64]$} \\
\hline full set & & Hetero & 545,247 & 211,425 & 175,487 & $O B O R^{\dagger}$ & $0.15[0.07,0.23]$ & $0.07[-0.07,0.22]$ & $0.14[0.00,0.36]$ & {$[-0.24,0.39]$} \\
\hline
\end{tabular}

Note. $\mathrm{k}=$ number of samples, $\# \mathrm{OB}=$ number of older brothers, $\# \mathrm{OS}=$ number of older sisters, $\mathrm{FEM}=$ estimated fixed-effect summary effect, REM = estimated random-effects summary effect, $\hat{\tau}=$ estimated standard deviation of population effects, $C I=95 \%$ Confidence Interval, $P I=95 \%$ prediction interval. ${ }^{*} \mathrm{k}=$ 59 (vs. 64) due to nonreporting of number of younger brothers and / or younger sisters in [62], [59], [65], [61], and [81]. ${ }^{\dagger} \mathrm{k}=14$ (vs. 17) due to nonreporting of number of younger brothers and / or sisters in [62], [57], and [81]. "-" = Redundant, as due to the REML estimate of $\hat{\imath}$ being 0 exactly, the prediction intervals and confidence intervals are numerically identical. 
Figures 

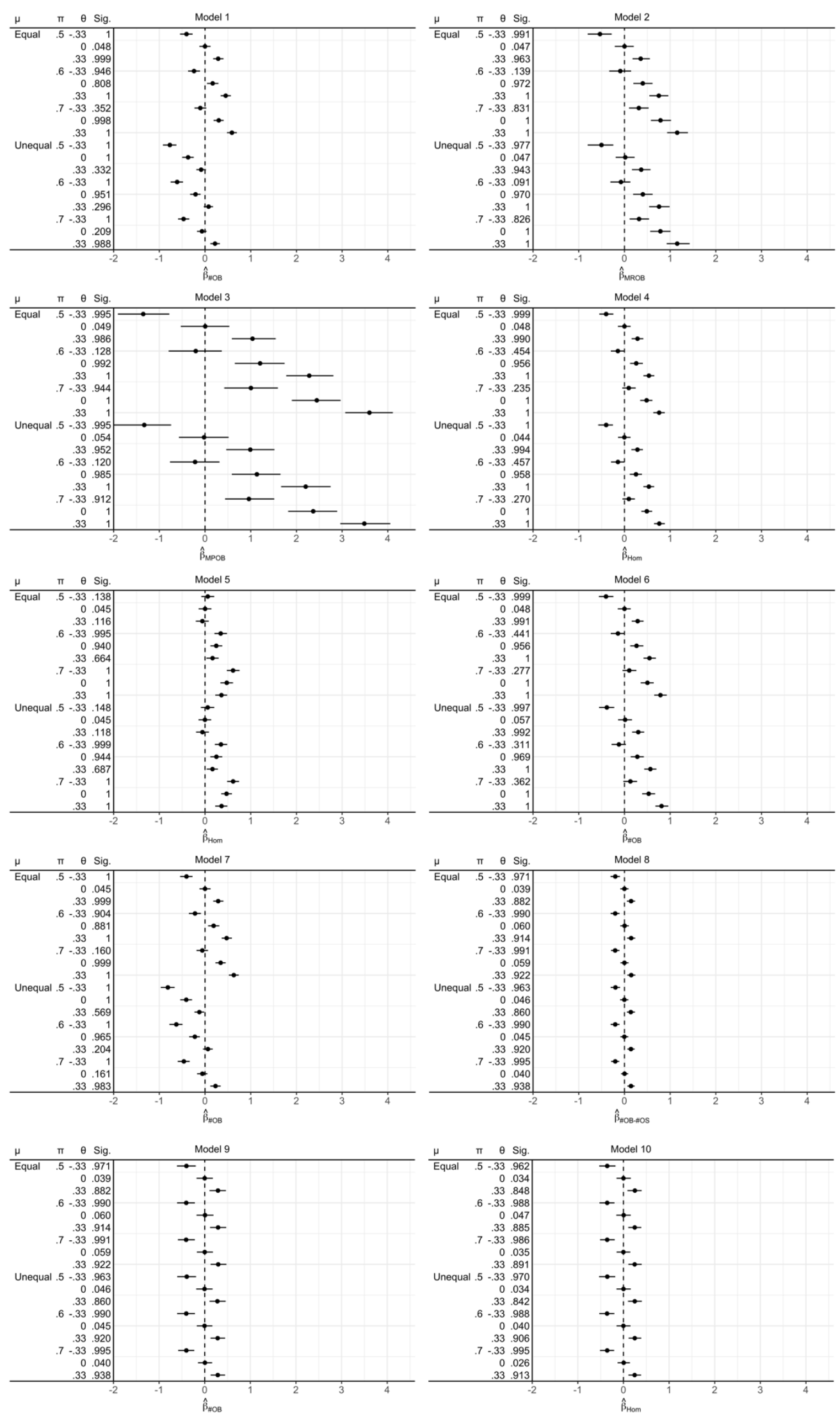
Figure 1. Results of the simulation study for Models 1 to 6. Points depict average estimates (horizontal axis) of the effect of interest in $\ln O R$ units over 1,000 replicates for each combination of $\mu, \pi$, and $\theta$ (vertical axis). The endpoints of the error bars depict the .025 and .975 quantiles of the estimate distributions. The table column Sig. lists the proportion of estimates with $p<.05$ (two-tailed). The table column $\mu$ indicates whether the mean number of all siblings was equal (i.e., 2.25) for both homosexual and heterosexual participants, or unequal (3.31 and 2.19, respectively). 


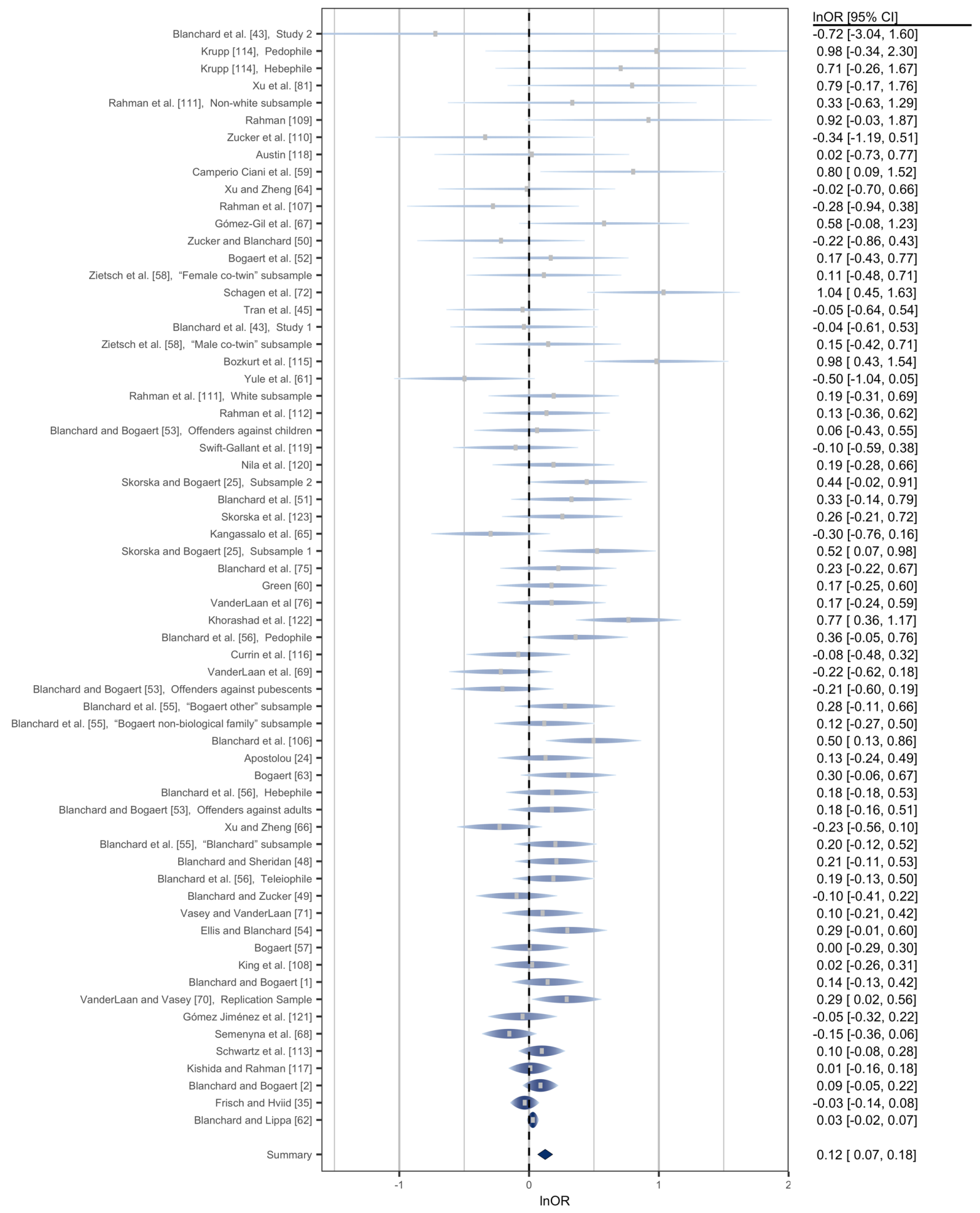

Figure 2. Rainforest plot of the random-effects meta-analysis of all male sample lnOR effect sizes. In this plot, the sample weights are both encoded in the degree of saturation and the thickness of the shaded regions, with heavier effect sizes being more saturated and thicker. The widths of the shaded regions correspond to $95 \%$ confidence intervals (CI); the point estimates are represented by the ticks inside these 
intervals. The teardrop-shape encodes the relative likelihood function of the mean of a normal distribution conditional on the standard deviation being equal to the observed standard error (i.e., the $\ln O R s$ are assumed to be distributed normally with known variance) and its horizontal mirror image over the range of the $95 \%$ CI. 


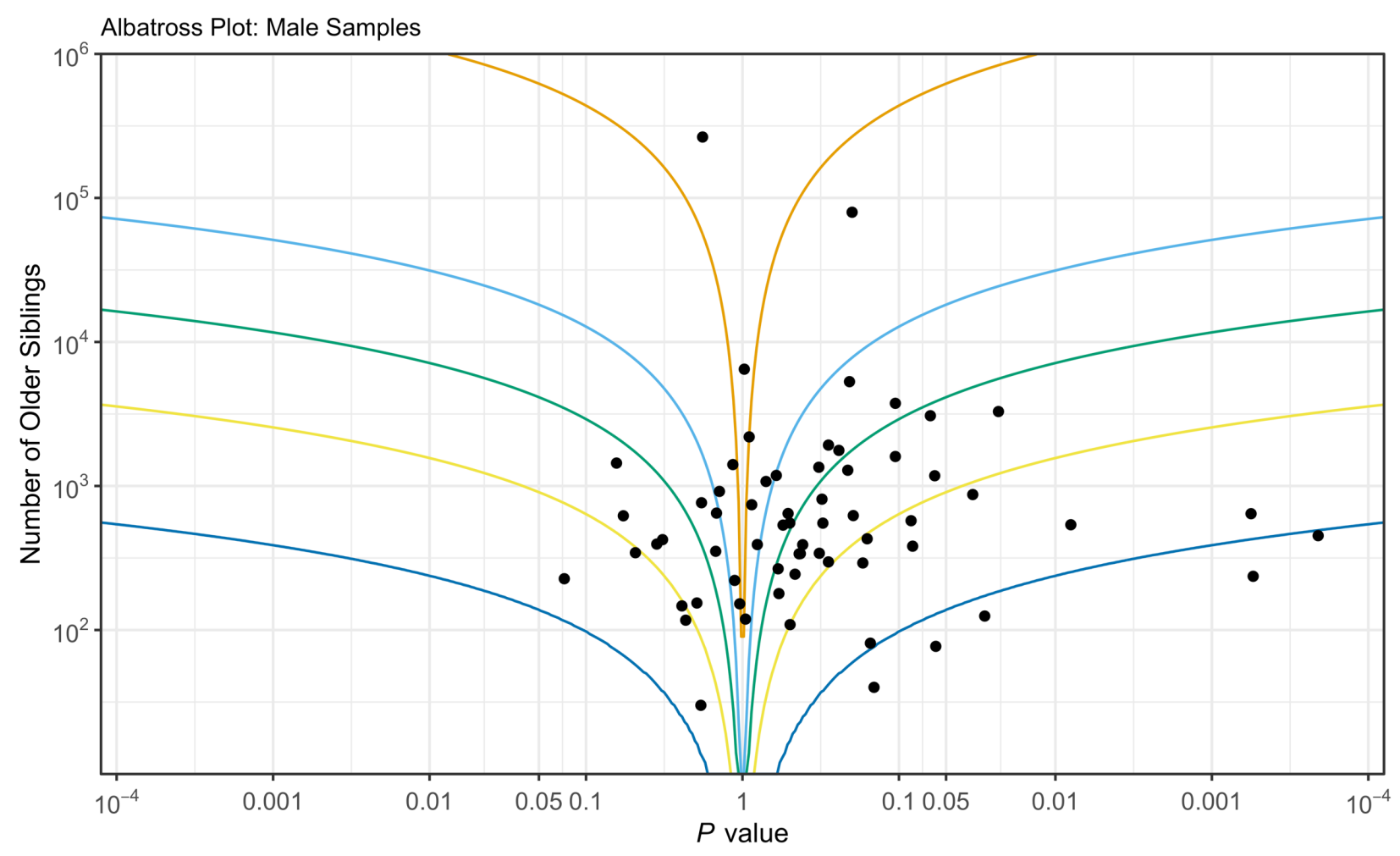

$$
\begin{aligned}
& \text { Negative Association Null Positive Association }
\end{aligned}
$$

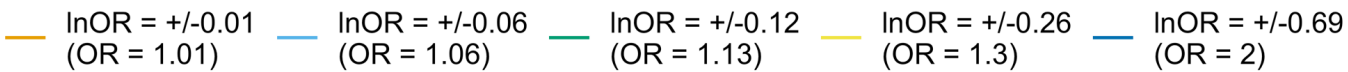

Figure 3 Albatross plot of all male sample $\ln O R$ effect sizes. The $x$-axis ( $p$ values) and the $y$-axis (total number of older siblings in each study) are on logarithmic scales. Effect sizes near a $p$ value of 1 indicate high compatibility between the observed effect and a model wherein the effect is $\ln O R=0$. The contours indicate effect ranges, which appeared plausible for the observed $p$ values. 


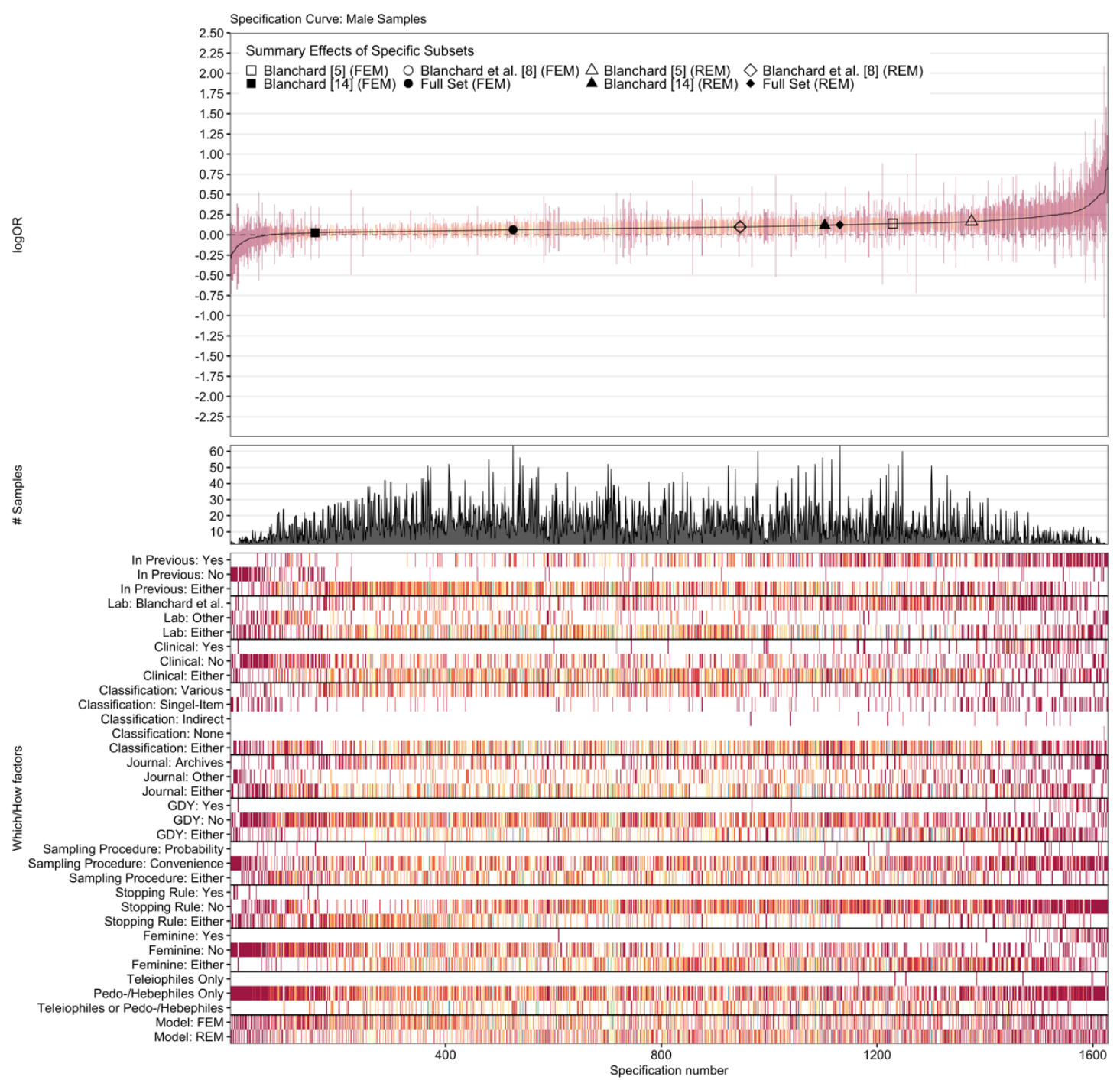

Figure 4 Specification-curve analysis of the male samples. From top to bottom, the panels encode each specification's estimated summary effect and 95\% confidence interval, number of samples included in the specifications (density plot), and the respective combination of factor levels (tile plot). The point estimates are arranged in ascending order and are connected by a black solid line, i.e., the specification curve. On this curve, the locations of previously published meta-analyses is highlighted. Each specification is colorcoded, using hues of six distinct colors (red, orange, yellow, green, blue, violet) on the spectrum from red to violet. A specification's color and hue are indicative of the number of samples included in the metaanalysis, with the number of samples increasing in the following order: red, orange, yellow, green, blue, violet. 


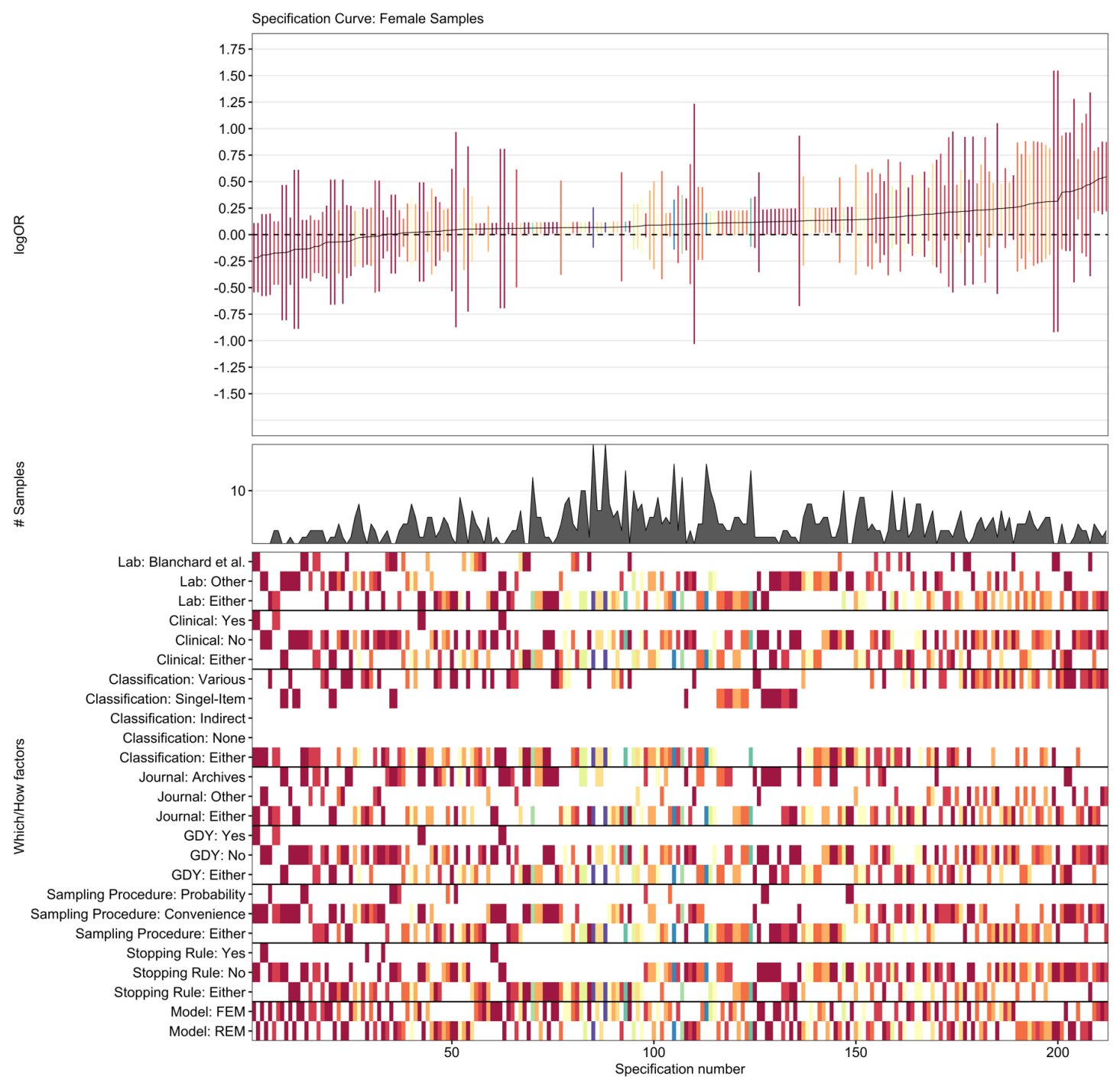

Figure 5 Specification-curve analysis of the female samples. From top to bottom, the panels encode each specification's estimated summary effect and $95 \%$ confidence interval, number of samples included in the specifications (density plot), and the respective combination of factor levels (tile plot). The point estimates are arranged in ascending order and are connected by a black solid line, i.e., the specification curve. Each specification is color-coded, using hues of six distinct colors (red, orange, yellow, green, blue, violet) on the spectrum from red to violet. A specification's color and hue are indicative of the number of samples included in the meta-analysis, with the number of samples increasing in the following order: red, orange, yellow, green, blue, violet. 
$S$ Values: Male Samples

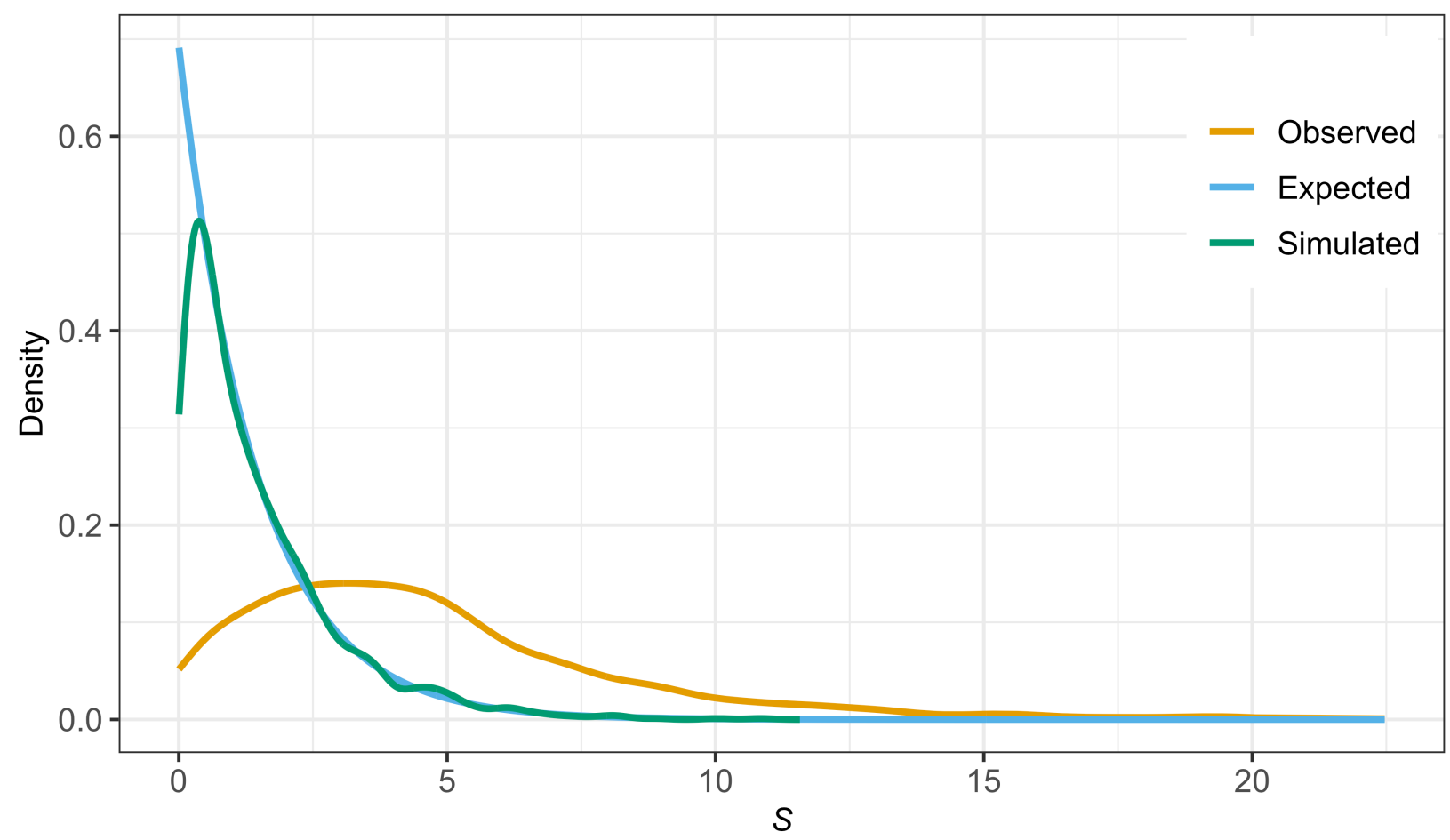

$S$ Values: Female Samples

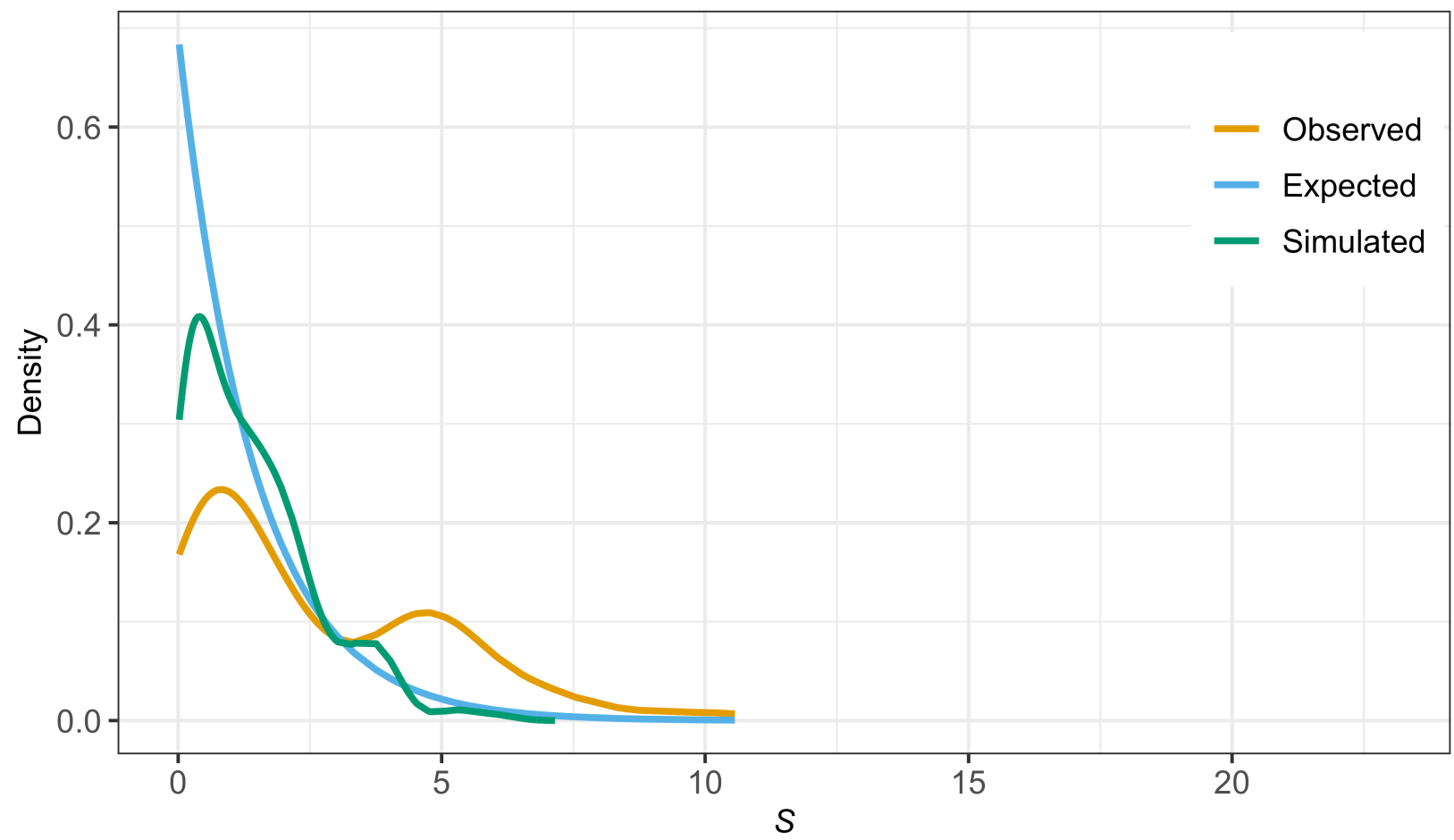

Figure 6 Kernel-density estimates of the S-values of the multiverses of meta-analytic evidence on the FBOE in all available data of a) male and b) female samples. The plots display observed S-values (orange line) alongside random values drawn from an Exponential $(\ln (2))$ distribution (simulated; green line) and the probability density function of an Exponential $(\ln (2))$ distribution (expected; blue line) for comparison. 